\title{
Warped flavor symmetry predictions for neutrino physics
}

\author{
Peng Chen, ${ }^{a}$ Gui-Jun Ding, ${ }^{a}$ Alma D. Rojas, ${ }^{b}$ C.A. Vaquera-Araujo ${ }^{b}$ and \\ J.W.F. Valle ${ }^{b, 1}$ \\ ${ }^{a}$ Department of Modern Physics, University of Science and Technology of China, \\ Hefei, Anhui 230026, China \\ ${ }^{b}$ AHEP Group, Institut de Física Corpuscular - C.S.I.C./Universitat de València, \\ Parc Cientific de Paterna, C/Catedratico José Beltrán, 2 E-46980 Paterna (València) - Spain \\ E-mail: pche@mail.ustc.edu.cn, dinggj@ustc.edu.cn, \\ alma.rojas@ific.uv.es, vaquera@ific.uv.es, valle@ific.uv.es
}

ABstract: A realistic five-dimensional warped scenario with all standard model fields propagating in the bulk is proposed. Mass hierarchies would in principle be accounted for by judicious choices of the bulk mass parameters, while fermion mixing angles are restricted by a $\Delta(27)$ flavor symmetry broken on the branes by flavon fields. The latter gives stringent predictions for the neutrino mixing parameters, and the Dirac CP violation phase, all described in terms of only two independent parameters at leading order. The scheme also gives an adequate CKM fit and should be testable within upcoming oscillation experiments.

KeYwords: Quark Masses and SM Parameters, Neutrino Physics, Discrete and Finite Symmetries

ARXIV EPRINT: 1509.06683

\footnotetext{
${ }^{1}$ URL: http://astroparticles.es/.
} 


\section{Contents}

1 Introduction $\quad 1$

2 Basic structure of the model $\quad 2$

3 Lepton sector $\quad 4$

3.1 Lepton masses and mixing 5

$\begin{array}{lll}3.2 & \text { Phenomenological implications } & 8\end{array}$

4 Quark sector $\quad 10$

5 High order corrections $\quad 14$

6 Conclusions 16

$\begin{array}{ll}\text { A The profile of the Higgs zero mode } & 17\end{array}$

$\begin{array}{ll}\text { B Group theory of } \Delta(27) \text { and its representation } & 18\end{array}$

$\begin{array}{ll}\text { C Vacuum alignment } & 21\end{array}$

\section{Introduction}

The understanding of flavor constitutes one of the most stubborn open challenges in particle physics [1]. Two aspects of the problem are the understanding of fermion mass hierarchies as well as mixing parameters. Various types of flavor symmetries have been invoked in this context [2-9]. These efforts have been partly motivated by the original success of the tribimaximal mixing ansatz [10]. The resulting non-Abelian flavor symmetries are typically broken spontaneously down to two different residual subgroups in the neutrino and the charged lepton sectors, leading to zero reactor mixing parameter, $\theta_{13}=0$. However, the measurement of a non-zero value for the reactor angle [11-14] implies the need to revamp the original flavor symmetry-based approaches in order to generate $\theta_{13} \neq 0$ [15] or else look for alternative possibilities, such as bi-large neutrino mixing [16-18].

The existence of warped extra dimensions has been advocated by Randall \& Sundrum [19] as a way to address the hierarchy problem, since the fundamental scale of gravity is exponentially reduced from the Planck mass down to the $\mathrm{TeV}$ scale as a result of having the Higgs sector localized near the boundary of the extra dimensions. Moreover, if standard model fermions are allowed to propagate in the bulk and also become localized towards either brane, the scenario can also address the flavor problem possibly acting in synergy with the flavor group predictions. This is what we do in the present paper. 
The idea of combining discrete flavor symmetries and extra dimensions is quite attractive and has already been discussed in the literature within the context of large extra dimensions [20-22], warped extra dimensions [23-31] and holographic composite Higgs models [32-34]. However, such models try to generate tri-bimaximal neutrino mixing, which has been ruled out by the measurement of the reactor angle $\theta_{13}$ [11-14] and also global fits of neutrino oscillation data [35]. One of us has constructed a warped extra dimension model with $S_{4}$ flavor symmetry where democratic mixing is produced at leading order and non-zero $\theta_{13}$ can arise from subleading corrections [36]. In this work, we shall re-consider the issue of predicting flavor properties in particle physics by combining the conventional predictive power inherent in the use of non-Abelian flavor symmetries with the presence of warped extra dimensions. We propose a warped five-dimensional scenario in which all matter fields propagate in the bulk and neutrinos are treated as Dirac particles. Our model can accommodate all the strengths of the standard model Yukawa couplings and resulting fermion mass hierarchies by making adequate choices of fermion bulk mass parameters, while the fermion mixing parameters can be restricted by means of the assumed flavor symmetry. We present a $\Delta(27)$ based flavor symmetry which nicely describes the neutrino oscillation parameters in terms of just two independent parameters, leading to interesting correlations involving the neutrino mass hierarchy and the leptonic Dirac CP phase, not yet reliably determined by current global oscillation fit [35]. Our predictions include a neat leading order relation between the solar and reactor mixing parameters which should be tested at future oscillation experiments.

\section{Basic structure of the model}

In this section we present the basic setup of a warped five-dimensional (5D) model for fermions, constructed under a $\Delta(27) \otimes Z_{4} \otimes Z_{4}^{\prime}$ flavor symmetry. The $5 \mathrm{D}$ field theory is defined on a slice of $\mathrm{AdS}_{5}$, where the bulk geometry is described by the metric

$$
d s^{2}=e^{-2 k y} \eta_{\mu \nu} d x^{\mu} d x^{\nu}-d y^{2},
$$

with $\eta_{\mu \nu}=\operatorname{diag}(1,-1,-1,-1)$ and $k$ as the $\operatorname{AdS}_{5}$ curvature scale. The fifth dimension $y$ is compactified on $S_{1} / Z_{2}$, and two flat 3-branes of opposite tension are attached to the orbifold fixed points, located at $y=0$ (UV brane) and $y=L$ (IR brane).

Our framework is built upon the most minimal version of the RS model. We adopt a non-custodial $G_{\mathrm{SM}}=\mathrm{SU}(2)_{L} \otimes \mathrm{U}(1)_{Y}$ bulk electroweak symmetry, where the $5 \mathrm{D}$ fermions and the Higgs field are allowed to propagate into the bulk. It is well known that models with a brane-localized Higgs and no custodial symmetry suffer from large constraints imposed by electroweak precision tests $[37,38]$. However, the tensions with electroweak precision tests [39-42] and flavour physics [42-45] can be significantly reduced in the case of a 5D Higgs field living in the bulk, offering a new elegant explanation for the the tiny neutrino masses [46, 47]. That is to say, a $\mathrm{SU}(2)_{L}$ doublet bulk Higgs field

$$
H=\left(\begin{array}{c}
\phi^{+} \\
\phi^{0}
\end{array}\right),
$$


is responsible for the spontaneous symmetry breaking (SSB) of $G_{\mathrm{SM}}$ to $\mathrm{U}(1)_{E M}$ through its vacuum expectation value (VEV), where the superscripts "+" and "0" denote the electric charge of the field. The 5D Higgs field $H\left(x^{\mu}, y\right)$ can be decomposed into Kaluza-Klein (KK) modes as

$$
H\left(x^{\mu}, y\right)=H\left(x^{\mu}\right) \frac{f_{H}(y)}{\sqrt{L}}+\text { heavy KK Modes } .
$$

For an adequate choice of the Higgs potential (see appendix A for an explicit realization), its zero mode profile $f_{H}(y)$ can be written as $[48,49]$

$$
f_{H}(y)=\sqrt{\frac{2 k L(1-\beta)}{1-e^{-2(1-\beta) k L}}} e^{k L} e^{(2-\beta) k(y-L)},
$$

where we have introduced the Higgs localization parameter $\beta=\sqrt{4+m_{H}^{2} / k^{2}}$ in terms of the Higgs field bulk mass parameter $m_{H}$. Furthermore, the Higgs zero mode obtains a VEV:

$$
\left\langle H\left(x^{\mu}\right)\right\rangle=\frac{v_{H}}{\sqrt{2}}\left(\begin{array}{l}
0 \\
1
\end{array}\right),
$$

and it is peaked toward the IR brane, allowing for electroweak SSB at the TeV scale. The fermion content is the same as in the standard model. Three families of fermion fields are required to describe each generation (labeled by $i=1,2,3$ ) of quarks and leptons. All fermion fields propagate into the bulk and transform under the minimal representation of the gauge group $\mathrm{SU}(2)_{L} \otimes \mathrm{U}(1)_{Y}$. In the lepton sector the three multiplets of the model are given as

$$
\Psi_{\ell_{i}}=\left(\begin{array}{c}
\nu_{i}^{[++]} \\
e_{i}^{[++]}
\end{array}\right) \sim(\mathbf{2},-1), \quad \Psi_{e_{i}}=e_{i}^{[--]} \sim(\mathbf{1},-2), \quad \Psi_{\nu_{i}}=\nu_{i}^{[--]} \sim(\mathbf{1}, 0),
$$

while for the quark sector we have

$$
\Psi_{Q_{i}}=\left(\begin{array}{c}
u_{i}^{[++]} \\
d_{i}^{[++]}
\end{array}\right) \sim(\mathbf{2}, 1 / 3), \quad \Psi_{d_{i}}=d_{i}^{[--]} \sim(\mathbf{1},-2 / 3), \quad \Psi_{u_{i}}=u_{i}^{[--]} \sim(\mathbf{1}, 4 / 3) .
$$

In the above equations, each 5D fermion field is described by a 4-component Dirac spinor field, and fields with different sign assignments must be understood as independent. The bracketed signs indicate Neumann $(+)$ or Dirichlet $(-)$ BCs for the left-handed component of the corresponding field, on both UV and IR branes. The right-handed part of the field satisfies opposite BCs. Only fields with $[++]$ BCs have left-handed zero modes, whereas right-handed zero modes exist solely for fields with [--] BCs. The KK decomposition for such fields has the form

$$
\begin{aligned}
& \psi^{[++]}\left(x^{\mu}, y\right)=\frac{e^{2 k y}}{\sqrt{L}}\left\{\psi_{L}\left(x^{\mu}\right) f_{L}^{(0)}\left(y, c_{L}\right)+\text { heavy KK modes }\right\}, \\
& \psi^{[--]}\left(x^{\mu}, y\right)=\frac{e^{2 k y}}{\sqrt{L}}\left\{\psi_{R}\left(x^{\mu}\right) f_{R}^{(0)}\left(y, c_{R}\right)+\text { heavy KK modes }\right\},
\end{aligned}
$$


with $\psi=\nu_{i}, e_{i}, u_{i}, d_{i}$, and zero mode profiles [50-52]

$$
f_{L}^{(0)}\left(y, c_{L}\right)=\sqrt{\frac{\left(1-2 c_{L}\right) k L}{e^{\left(1-2 c_{L}\right) k L}-1}} e^{-c_{L} k y}, \quad f_{R}^{(0)}\left(y, c_{R}\right)=\sqrt{\frac{\left(1+2 c_{R}\right) k L}{e^{\left(1+2 c_{R}\right) k L}-1}} e^{c_{R} k y}
$$

where $c_{L}$ and $c_{R}$ are the bulk mass parameters of the $5 \mathrm{D}$ fermion fields in units of the $\mathrm{AdS}_{5}$ curvature $k$. Thus, the low energy spectrum contains left-handed doublets $\ell_{i L}=\left(\nu_{i L}, e_{i L}\right)$, $Q_{i L}=\left(u_{i L}, d_{i L}\right)$, alongside right-handed singlets $\nu_{i R}, e_{i R}, u_{i R}, d_{i R}$. In the following, we identify all standard model fields with this set of zero modes (i.e. the so called zero mode approximation, ZMA). For future convenience, we denote the flavor components of charged leptons and quarks as $e_{1,2,3}=e, \mu, \tau ; Q_{1,2,3}=U, C, T ; u_{1,2,3}=u, c, t ; d_{1,2,3}=d, s, b$.

In the present work, we choose the flavor symmetry to be $\Delta(27)$, augmented by the auxiliary symmetry $Z_{4} \otimes Z_{4}^{\prime}$. The group $\Delta(27)$ was originally proposed to explain the fermion masses and flavor mixing in refs. [53, 54], and has been used for Dirac neutrinos in [55] by one of us. Here we study its implementation in a warped extra dimensional theory. The flavor symmetry $\Delta(27) \otimes Z_{4} \otimes Z_{4}^{\prime}$ is broken by brane localized flavons, transforming as singlets under $G_{\mathrm{SM}}$. We introduce a set of flavons $\xi, \sigma_{1}, \sigma_{2}$ localized on the IR brane, and a flavon $\varphi$ localized on the UV brane. Both $\xi$ and $\varphi$ are assigned to the three-dimensional representation 3 of $\Delta(27)$, while $\sigma_{1}$ and $\sigma_{2}$ transform as inequivalent one-dimensional representations $\mathbf{1}_{0,1}$ and $\mathbf{1}_{0,0}$ respectively. A summary of the $\Delta(27)$ group properties and its representations can be found in appendix B. There are two different scenarios for the model, determined by the two possible VEV alignments for $\xi$, namely:

$$
\begin{array}{ll}
\langle\xi\rangle=(0,1,0) v_{\xi}, & \text { Case I, } \\
\langle\xi\rangle=(1, \omega, 1) v_{\xi}, & \text { Case II, }
\end{array}
$$

with $\omega=e^{2 \pi i / 3}$. As indicated above, we will denote the models described by each alignment as cases I and II, respectively. Note that the case II vacuum pattern frequently appears in the context of geometrical CP violation $[56,57]$. The VEVs for the remaining flavon fields are

$$
\langle\varphi\rangle=(1,1,1) v_{\varphi}, \quad\left\langle\sigma_{1}\right\rangle=v_{\sigma_{1}}, \quad\left\langle\sigma_{2}\right\rangle=v_{\sigma_{2}}
$$

Further details regarding this vacuum configuration are offered in appendix C.

\section{Lepton sector}

Once the basic framework has been laid out, we are in position to discuss the structure of the lepton sector and its phenomenological implications. As we will show below, charged lepton as well as Dirac neutrino masses are generated at leading order (LO), and non-zero values for the "reactor angle" $\theta_{13}$ arise naturally. The model is predictive, in the sense that the three mixing angles and the Dirac CP phase will ultimately be determined in terms of only two parameters. 


\begin{tabular}{|c|c|c|c|c|c|c|c|c|c|c|c|c|}
\hline Field & $\Psi_{\ell}$ & $\Psi_{e}$ & $\Psi_{\mu}$ & $\Psi_{\tau}$ & $\Psi_{\nu_{1}}$ & $\Psi_{\nu_{2}}$ & $\Psi_{\nu_{3}}$ & $H$ & $\varphi$ & $\xi$ & $\sigma_{1}$ & $\sigma_{2}$ \\
\hline$\Delta(27)$ & $\mathbf{3}$ & $\mathbf{1}_{0,0}$ & $\mathbf{1}_{1,0}$ & $\mathbf{1}_{2,0}$ & $\mathbf{1}_{0,0}$ & $\mathbf{1}_{0,0}$ & $\mathbf{1}_{0,0}$ & $\mathbf{1}_{0,0}$ & $\mathbf{3}$ & $\mathbf{3}$ & $\mathbf{1}_{0,1}$ & $\mathbf{1}_{0,0}$ \\
\hline$Z_{4}$ & 1 & 1 & 1 & 1 & -1 & $i$ & -1 & 1 & 1 & -1 & 1 & $i$ \\
\hline$Z_{4}^{\prime}$ & 1 & $i$ & $i$ & $i$ & -1 & -1 & -1 & 1 & $-i$ & 1 & -1 & -1 \\
\hline
\end{tabular}

Table 1. Particle content and transformation properties of the lepton and scalar sectors under the flavor symmetry $\Delta(27) \otimes Z_{4} \otimes Z_{4}^{\prime}$.

\subsection{Lepton masses and mixing}

The transformation properties of leptons and scalars under the family symmetry $\Delta(27) \otimes$ $Z_{4} \otimes Z_{4}^{\prime}$ are given in table 1 . Note that the Higgs field is inert under the flavor symmetry. Since the three left-handed lepton doublets are unified into a faithful triplet $\mathbf{3}$ of $\Delta(27)$, they will share one common bulk mass parameter $c_{\ell}$. On the other hand, both righthanded charged leptons and right-handed neutrinos are assigned to singlet representations of $\Delta(27)$. Therefore, there are six different bulk mass parameters $c_{e_{i}}$ and $c_{\nu_{i}}(i=1,2,3)$ for these fields. From the particle transformation properties we can write the most general lepton Yukawa interactions that are both gauge and flavor invariant at LO: ${ }^{1}$

$$
\begin{aligned}
\mathcal{L}_{Y}^{l}= & \frac{\sqrt{G}}{\Lambda^{\frac{5}{2}}}\left\{y_{e}\left(\varphi \bar{\Psi}_{\ell}\right)_{\mathbf{1}_{0,0}} H \Psi_{e}+y_{\mu}\left(\varphi \bar{\Psi}_{\ell}\right)_{\mathbf{1}_{2,0}} H \Psi_{\mu}+y_{\tau}\left(\varphi \bar{\Psi}_{\ell}\right)_{\mathbf{1}_{1,0}} H \Psi_{\tau}\right\} \delta(y) \\
& +\frac{\sqrt{G}}{\left(\Lambda^{\prime}\right)^{\frac{7}{2}}}\left\{y_{11}\left(\xi \sigma_{1} \bar{\Psi}_{\ell}\right)_{\mathbf{1}_{0,0}} \widetilde{H} \Psi_{\nu_{1}}+y_{31}\left(\xi \sigma_{1}^{*} \bar{\Psi}_{\ell}\right)_{\mathbf{1}_{0,0}} \widetilde{H} \Psi_{\nu_{1}}+y_{22}\left(\xi \sigma_{2} \bar{\Psi}_{\ell}\right)_{\mathbf{1}_{0,0}} \widetilde{H} \Psi_{\nu_{2}}\right. \\
& \left.\quad+y_{13}\left(\xi \sigma_{1} \bar{\Psi}_{\ell}\right)_{\mathbf{1}_{0,0}} \widetilde{H} \Psi_{\nu_{3}}+y_{33}\left(\xi \sigma_{1}^{*} \bar{\Psi}_{\ell}\right)_{\mathbf{1}_{0,0}} \widetilde{H} \Psi_{\nu_{3}}\right\} \delta(y-L)+\text { h.c. }
\end{aligned}
$$

with $\widetilde{H} \equiv i \tau_{2} H^{*}$, and $\tau_{i}$ as the Pauli matrices. After electroweak and flavor spontaneous symmetry breaking, all leptons develop masses dictated by the above Yukawa interactions. The generated masses are modulated by the overlap of the relevant zero mode fermion profiles, the VEV profile of the Higgs, and the flavon VEVs given in eqs. (2.10), (2.11).

From eq. (3.1), The mass matrix $m_{l}$ for charged leptons is

$$
m_{l}=\frac{1}{(L \Lambda)^{\frac{3}{2}}} \frac{v_{\varphi}}{\Lambda} \frac{v_{H}}{\sqrt{2}} \sqrt{3} U_{l}\left(\begin{array}{ccc}
\widetilde{y}_{e} & 0 & 0 \\
0 & \widetilde{y}_{\mu} & 0 \\
0 & 0 & \widetilde{y}_{\tau}
\end{array}\right)
$$

where $U_{l}$ stands for the so-called magic matrix

$$
U_{l}=\frac{1}{\sqrt{3}}\left(\begin{array}{ccc}
1 & 1 & 1 \\
1 & \omega & \omega^{2} \\
1 & \omega^{2} & \omega
\end{array}\right)
$$

\footnotetext{
${ }^{1}$ Notice that the Majorana coupling of the form $\bar{\Psi}_{\nu_{i}} \Psi_{\nu_{j}}^{c}$ with $i, j=1,3$ can be forbidden by including addtional auxiliary cyclic group such as $Z_{3}$.
} 
and $\widetilde{y}_{e, \mu, \tau}$ are modified Yukawa couplings defined as

$$
\widetilde{y}_{e, \mu, \tau}=y_{e, \mu, \tau} F\left(0, c_{\ell}, c_{e_{i}}\right),
$$

in terms of the overlapping function

$$
\begin{aligned}
& F\left(y, c_{L}, c_{R}\right) \equiv f_{L}^{(0)}\left(y, c_{L}\right) f_{R}^{(0)}\left(y, c_{R}\right) f_{H}(y) \\
& =\sqrt{\frac{2\left(1-\beta_{H}\right)\left(1-2 c_{L}\right)\left(1+2 c_{R}\right) k^{3} L^{3}}{\left[1-e^{-2\left(1-\beta_{H}\right) k L}\right]\left[e^{\left(1-2 c_{L}\right) k L}-1\right]\left[e^{\left(1+2 c_{R}\right) k L}-1\right]}} e^{-\left(1-\beta_{H}\right) k L} e^{\left(2-\beta_{H}-c_{L}+c_{R}\right) k y} .
\end{aligned}
$$

Given that $U_{l}^{\dagger} U_{l}=1$, the diagonalization of the charged lepton mass matrix is straightforward, leading to charged lepton masses of the form

$$
m_{e, \mu, \tau}=\frac{\sqrt{3} \widetilde{y}_{e, \mu, \tau}}{(L \Lambda)^{\frac{3}{2}}} \frac{v_{\varphi}}{\Lambda} \frac{v_{H}}{\sqrt{2}} .
$$

Analogously, taking into account the two distinct VEV alignments for the flavon triplet $\xi$ in eq. (2.10), the neutrino mass matrix for each respective case can be written as

$$
\begin{aligned}
& m_{\nu}^{\mathrm{I}}=\frac{1}{\left(L \Lambda^{\prime}\right)^{\frac{3}{2}}} \frac{v_{\xi}}{\Lambda^{\prime}} \frac{v_{H}}{\sqrt{2}}\left(\begin{array}{ccc}
\widetilde{y}_{11} \frac{v_{\sigma_{1}}}{\Lambda^{\prime}} & 0 & \widetilde{y}_{13} \frac{v_{\sigma_{1}}}{\Lambda^{\prime}} \\
0 & \widetilde{y}_{22} \frac{v_{\sigma_{2}}}{\Lambda^{\prime}} & 0 \\
\widetilde{y}_{31} \frac{v_{\sigma_{1}}^{*}}{\Lambda^{\prime}} & 0 & \widetilde{y}_{33} \frac{v_{\sigma_{1}}^{*}}{\Lambda^{\prime}}
\end{array}\right), \\
& m_{\nu}^{\mathrm{II}}=\frac{1}{\left(L \Lambda^{\prime}\right)^{\frac{3}{2}}} \frac{v_{\xi}}{\Lambda^{\prime}} \frac{v_{H}}{\sqrt{2}} \sqrt{3} V_{0}\left(\begin{array}{ccc}
\widetilde{y}_{11} \frac{v_{\sigma_{1}}}{\Lambda^{\prime}} & 0 & \widetilde{y}_{13} \frac{v_{\sigma_{1}}}{\Lambda^{\prime}} \\
0 & \widetilde{y}_{22} \frac{v_{\sigma_{2}}}{\Lambda^{\prime}} & 0 \\
\widetilde{y}_{31} \frac{v_{\sigma_{1}}^{*}}{\Lambda^{\prime}} & 0 & \widetilde{y}_{33} \frac{v_{\sigma_{1}}^{*}}{\Lambda^{\prime}}
\end{array}\right),
\end{aligned}
$$

with

$$
\widetilde{y}_{i j}=y_{i j} F\left(L, c_{\ell}, c_{\nu_{j}}\right)
$$

and

$$
V_{0} \equiv \frac{1}{\sqrt{3}}\left(\begin{array}{ccc}
\omega & 1 & 1 \\
1 & \omega & 1 \\
1 & 1 & \omega
\end{array}\right) .
$$

Thus, the diagonalizing matrix for the neutrino sector can be parameterized as

$$
\begin{aligned}
U_{\nu}^{\mathrm{I}} & =\left(\begin{array}{ccc}
\cos \theta_{\nu} & 0 & \sin \theta_{\nu} e^{i \varphi_{\nu}} \\
0 & 1 & 0 \\
-\sin \theta_{\nu} e^{-i \varphi_{\nu}} & 0 & \cos \theta_{\nu}
\end{array}\right), \\
U_{\nu}^{\mathrm{II}} & =V_{0}\left(\begin{array}{ccc}
\cos \theta_{\nu} & 0 \sin \theta_{\nu} e^{i \varphi_{\nu}} \\
0 & 1 & 0 \\
-\sin \theta_{\nu} e^{-i \varphi_{\nu}} & 0 & \cos \theta_{\nu}
\end{array}\right) .
\end{aligned}
$$

In terms of the auxiliary functions

$$
X_{\nu}^{ \pm}=\left|\widetilde{y}_{31}\right|^{2}+\left|\widetilde{y}_{33}\right|^{2} \pm\left|\widetilde{y}_{11}\right|^{2} \pm\left|\widetilde{y}_{13}\right|^{2}, \quad Y_{\nu}=\widetilde{y}_{11} \widetilde{y}_{33}-\widetilde{y}_{13}^{*} \widetilde{y}_{31}^{*}, \quad Z_{\nu}=\widetilde{y}_{11} \widetilde{y}_{31}^{*}+\widetilde{y}_{13} \widetilde{y}_{33}^{*},
$$


the relevant parameters of the model, $\theta_{\nu}$ and $\varphi_{\nu}$, are given by

$$
\tan 2 \theta_{\nu}=2\left|Z_{\nu}\right| / X_{\nu}^{-}, \quad \varphi_{\nu}=\arg \left(v_{\sigma_{1}}^{2} Z_{\nu}\right),
$$

and the neutrino mass eigenvalues for both $\mathrm{NH}$ and $\mathrm{IH}$ are determined as

- Case I

$\mathrm{NH}: \quad m_{1}=\frac{\widetilde{v}_{1}}{\sqrt{2}} M^{-}\left(X_{\nu}^{+}, Y_{\nu}\right), \quad m_{2}=\widetilde{v}_{2}\left|\widetilde{y}_{22}\right|, \quad m_{3}=\frac{\widetilde{v}_{1}}{\sqrt{2}} M^{+}\left(X_{\nu}^{+}, Y_{\nu}\right), \quad$ for $X_{\nu}^{-} \cos 2 \theta_{\nu}>0$,

$\mathrm{IH}: \quad m_{1}=\frac{\widetilde{v}_{1}}{\sqrt{2}} M^{+}\left(X_{\nu}^{+}, Y_{\nu}\right), \quad m_{2}=\widetilde{v}_{2}\left|\widetilde{y}_{22}\right|, \quad m_{3}=\frac{\widetilde{v}_{1}}{\sqrt{2}} M^{-}\left(X_{\nu}^{+}, Y_{\nu}\right), \quad$ for $X_{\nu}^{-} \cos 2 \theta_{\nu}<0$,

- Case II

$\mathrm{NH}: \quad m_{1}=\sqrt{\frac{3}{2}} \widetilde{v}_{1} M^{-}\left(X_{\nu}^{+}, Y_{\nu}\right), \quad m_{2}=\sqrt{3} \widetilde{v}_{2}\left|\widetilde{y}_{22}\right|, \quad m_{3}=\sqrt{\frac{3}{2}} \widetilde{v}_{1} M^{+}\left(X_{\nu}^{+}, Y_{\nu}\right), \quad$ for $X_{\nu}^{-} \cos 2 \theta_{\nu}>0$,

$\mathrm{IH}: \quad m_{1}=\sqrt{\frac{3}{2}} \widetilde{v}_{1} M^{+}\left(X_{\nu}^{+}, Y_{\nu}\right), \quad m_{2}=\sqrt{3} \widetilde{v}_{2}\left|\widetilde{y}_{22}\right|, \quad m_{3}=\sqrt{\frac{3}{2}} \widetilde{v}_{1} M^{-}\left(X_{\nu}^{+}, Y_{\nu}\right), \quad$ for $X_{\nu}^{-} \cos 2 \theta_{\nu}<0$,

where we have defined

$$
M^{ \pm}(x, y)=\sqrt{x \pm \sqrt{x^{2}-4|y|^{2}}}
$$

and

$$
\widetilde{v}_{\alpha}=\left|\frac{1}{\left(L \Lambda^{\prime}\right)^{\frac{3}{2}}} \frac{v_{\xi}}{\Lambda^{\prime}} \frac{v_{\sigma_{\alpha}}}{\Lambda^{\prime}} \frac{v_{H}}{\sqrt{2}}\right|, \quad \alpha=1,2 .
$$

Without loss of generality, the angle $\theta_{\nu}$ is restricted to the interval $[0, \pi]$. Notice that $X_{\nu}^{-} \cos 2 \theta_{\nu}=2\left|Z_{\nu}\right| \cos ^{2} 2 \theta_{\nu} / \sin 2 \theta_{\nu}$. As a result, for non-vanishing values of $Z_{\nu}$, the neutrino mass spectrum displays Normal Hierarchy (NH) provided $0<\theta_{\nu}<\pi / 2$, whereas Inverted Hierarchy (IH) is realized for $\pi / 2<\theta_{\nu}<\pi$. The angle $\varphi_{\nu}$, on the other hand, can take any value in the interval $[0,2 \pi]$.

At leading order, the lepton mixing matrix $U_{\mathrm{PMNS}}=U_{l}^{\dagger} U_{\nu}$ becomes

$$
\begin{aligned}
U_{\mathrm{PMNS}}^{\mathrm{I}} & =\frac{1}{\sqrt{3}}\left(\begin{array}{ccc}
\cos \theta_{\nu}-e^{-i \varphi_{\nu}} \sin \theta_{\nu} & 1 & \cos \theta_{\nu}+e^{i \varphi_{\nu}} \sin \theta_{\nu} \\
\cos \theta_{\nu}-\omega e^{-i \varphi_{\nu}} \sin \theta_{\nu} & \omega^{2} & \omega \cos \theta_{\nu}+e^{i \varphi_{\nu}} \sin \theta_{\nu} \\
\cos \theta_{\nu}-\omega^{2} e^{-i \varphi_{\nu}} \sin \theta_{\nu} & \omega & \omega^{2} \cos \theta_{\nu}+e^{i \varphi_{\nu}} \sin \theta_{\nu}
\end{array}\right), \\
U_{\mathrm{PMNS}}^{\mathrm{II}} & =\frac{-i \omega}{\sqrt{3}}\left(\begin{array}{ccc}
\cos \theta_{\nu}-e^{-i \varphi_{\nu}} \sin \theta_{\nu} & 1 & \cos \theta_{\nu}+e^{i \varphi_{\nu}} \sin \theta_{\nu} \\
\omega \cos \theta_{\nu}-\omega^{2} e^{-i \varphi_{\nu}} \sin \theta_{\nu} & 1 & \omega^{2} \cos \theta_{\nu}+\omega e^{i \varphi_{\nu}} \sin \theta_{\nu} \\
\omega \cos \theta_{\nu}-e^{-i \varphi_{\nu}} \sin \theta_{\nu} & \omega^{2} & \cos \theta_{\nu}+\omega e^{i \varphi_{\nu}} \sin \theta_{\nu}
\end{array}\right) .
\end{aligned}
$$

In both cases, the solar, atmospheric and reactor angles can be written in terms of $\theta_{\nu}$ and $\varphi_{\nu}$ as

$$
\sin ^{2} \theta_{12}=\frac{1}{2-\sin 2 \theta_{\nu} \cos \varphi_{\nu}}
$$


$\mathrm{NH}$

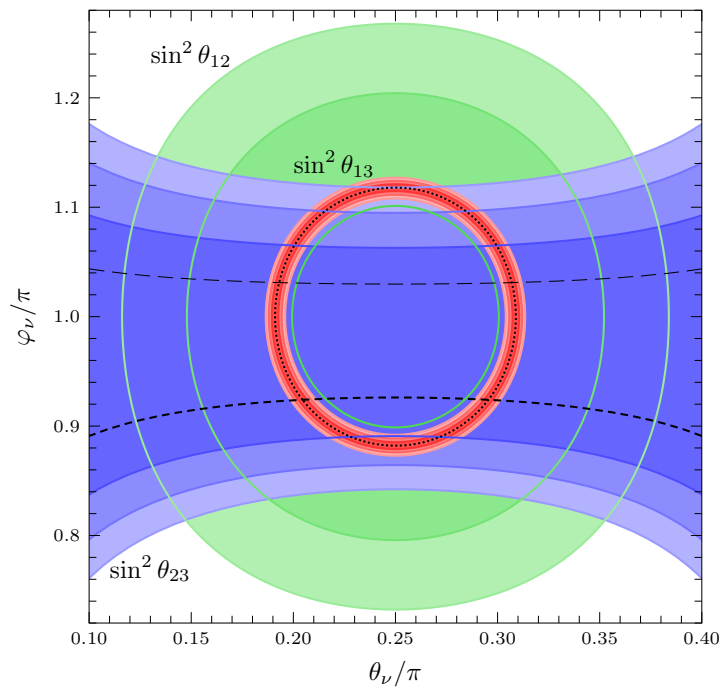

$\mathrm{IH}$

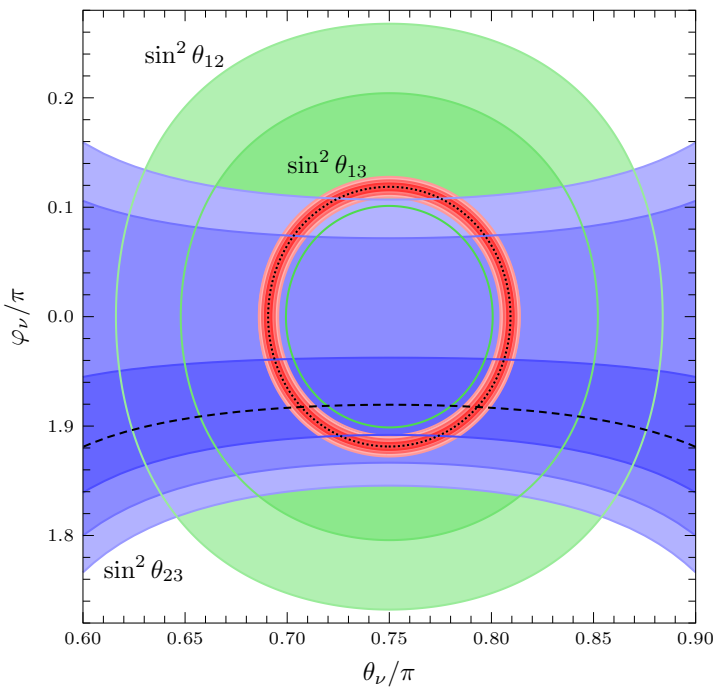

Figure 1. $1 \sigma, 2 \sigma$ and $3 \sigma$ ranges of $\sin ^{2} \theta_{12}$ (green), $\sin ^{2} \theta_{23}$ (blue) and $\sin ^{2} \theta_{13}$ (red) for normal (left panel) and inverted (right panel) neutrino mass hierarchies. Best-fit contours for $\sin ^{2} \theta_{13}\left(\sin ^{2} \theta_{23}\right)$ are indicated by dotted (short-dashed) lines. The long-dashed contour in the left panel represents the local minimum in the first octant of $\theta_{23}$.

$$
\begin{aligned}
& \sin ^{2} \theta_{23}=\frac{1-\sin 2 \theta_{\nu} \sin \left(\pi / 6-\varphi_{\nu}\right)}{2-\sin 2 \theta_{\nu} \cos \varphi_{\nu}} \\
& \sin ^{2} \theta_{13}=\frac{1}{3}\left(1+\sin 2 \theta_{\nu} \cos \varphi_{\nu}\right)
\end{aligned}
$$

A convenient description for the CP violating phase in this sector is the Jarlskog invariant $J_{\mathrm{CP}}=\operatorname{Im}\left[U_{e 1}^{*} U_{\mu 3}^{*} U_{\mu 1} U_{e 3}\right][58]$, which in this parameterization takes the compact form

$$
J_{\mathrm{CP}}=-\frac{1}{6 \sqrt{3}} \cos 2 \theta_{\nu}
$$

It is worthy of attention the independence of $J_{\mathrm{CP}}$ upon $\varphi_{\nu}$, and the simple predicted relation between the solar and reactor angles $\theta_{12}$ and $\theta_{13}$ :

$$
\sin ^{2} \theta_{12} \cos ^{2} \theta_{13}=\frac{1}{3} .
$$

\subsection{Phenomenological implications}

As shown above, only two parameters are required to generate the three angles and the Dirac CP violating phase characterizing the lepton mixing matrix, making this model highly predictive. In the remaining part of this section we explore in detail the predictions for the lepton mixing parameters and the neutrino mass spectrum.

In figure 1 , the $\theta_{\nu}-\varphi_{\nu}$ parameter region compatible with experimental data is delimited using the global fit of neutrino oscillations given in [35] for each mass ordering, shown as the left and right hand panel. The model can reproduce successfully the best-fit values for the atmospheric and reactor angles, reaching simultaneously the $2 \sigma$ region for the solar 


\begin{tabular}{|c|c|c|c|}
\hline & $\mathrm{NH}_{1}$ & $\mathrm{NH}_{2}$ & $\mathrm{IH}$ \\
\hline $\sin ^{2} \theta_{23} / 10^{-1}$ & 5.67 & 4.73 & 5.73 \\
\hline $\sin ^{2} \theta_{13} / 10^{-2}$ & 2.26 & 2.26 & 2.29 \\
\hline $\sin ^{2} \theta_{12} / 10^{-1}$ & 3.41 & 3.41 & 3.41 \\
\hline$J_{\mathrm{CP}} / 10^{-2}$ & $-(+) 2.71$ & $-(+) 3.37$ & $+(-) 2.57$ \\
\hline
\end{tabular}

Table 2. Central predictions for $\sin ^{2} \theta_{12}$ and $J_{\mathrm{CP}}$ obtained from the central values of the atmospheric and reactor angles reported in ref. [35]. The sign of $J_{C P}$ in the parentheses corresponds to the bracketed prediction for $\theta_{\nu}$ in eq. (3.26).

angle. The intersecting points of the "central" or best fit curve in the $\sin ^{2} \theta_{13}$ contour and the corresponding ones in the $\sin ^{2} \theta_{23}$ contour are located at

$$
\begin{array}{lll}
\mathrm{NH}_{1}: & \theta_{\nu} / \pi=0.204(0.296), & \varphi_{\nu} / \pi=0.924, \\
\mathrm{NH}_{2}: & \theta_{\nu} / \pi=0.193(0.307), & \varphi_{\nu} / \pi=1.031, \\
\mathrm{IH}: & \theta_{\nu} / \pi=0.707(0.793), & \varphi_{\nu} / \pi=1.917,
\end{array}
$$

where $\mathrm{NH}_{1}$ denotes the best-fit contour of $\sin ^{2} \theta_{23}$, and $\mathrm{NH}_{2}$ corresponds to its local minimum in the first octant. Notice that the numbers in parenthesis denote the intersection values within the range $\theta_{\nu} \in[\pi / 4, \pi / 2] \cup[3 \pi / 4, \pi]$.

Once we have determined $\theta_{\nu}$ and $\varphi_{\nu}$ from the central values of the atmospheric and reactor oscillation global fits, the predictions for the solar angle and the Jarlskog invariant can be straightforwardly obtained using eqs. (3.23), (3.24). For completeness, in table 2 we present the full set of mixing parameters derived from the points defined in eq. (3.26).

Remarkably, the central prediction for $\sin ^{2} \theta_{12}$ falls very close to its $1 \sigma$ boundary. In addition, notice that the $1 \sigma$ range of $J_{\mathrm{CP}}$ is entirely contained in the region $\theta_{\nu} \in$ $[0, \pi / 4] \cup[3 \pi / 4, \pi]$.

We conclude this section bringing forth a consistent realization of lepton masses and mixing angles. In the numerical analysis, we assume that the fundamental $5 \mathrm{D}$ scale is $k \simeq \Lambda \simeq M_{\mathrm{Pl}}$, with $M_{\mathrm{Pl}} \simeq 2.44 \times 10^{18} \mathrm{GeV}$ as the reduced Planck mass. We also set the scale $\Lambda^{\prime} \simeq k^{\prime}=k e^{-k L} \simeq 1.5 \mathrm{TeV}$ in order to account for the hierarchy between the Planck and the electroweak scales, allowing for the lowest KK gauge boson resonances (with masses $m_{K K}=$ $3 \sim 4 \mathrm{TeV})$ to be within the reach of the LHC experiments. The Higgs VEV is identified with its standard model value $v_{H} \simeq 246 \mathrm{GeV}$, and the ratios $v_{\varphi} / \Lambda, v_{\xi} / \Lambda^{\prime}, v_{\sigma_{1}} / \Lambda^{\prime}, v_{\sigma_{2}} / \Lambda^{\prime}$, are all fixed to 0.1 (thus considering real-valued flavon VEVs). The Higgs localization parameter $\beta$, common to all mass matrix elements, is chosen as 0.95 in the following discussion.

As an as illustrative example, we can choose $c_{\ell}=1.85, c_{e}=-0.27, c_{\mu}=-0.44$, $c_{\tau}=-0.71,\left|y_{e}\right|=0.861,\left|y_{\mu}\right|=0.898,\left|y_{\tau}\right|=0.994$ to generate the charged lepton masses $m_{e}=0.511 \mathrm{MeV}, m_{\mu}=105.7 \mathrm{MeV}, m_{\tau}=1.777 \mathrm{GeV}$. For the neutrino sector, benchmark points (BPs) in parameter space are given in table 3. There, the four BPs are labeled according to their hierarchy scheme and case as NH-I, NH-II, IH-I, IH-II. One sees that, indeed, the large disparity between charged lepton masses is reproduced for Yukawa couplings of the same order of magnitude. 


\begin{tabular}{|c|c|c|c|c|}
\hline & NH-I & NH-II & IH-I & IH-II \\
\hline$c_{\nu_{1}}$ & -1.40 & -1.41 & -1.39 & -1.40 \\
\hline$c_{\nu_{2}}$ & -1.38 & -1.40 & -1.33 & -1.35 \\
\hline$c_{\nu_{3}}$ & -1.34 & -1.36 & -1.34 & -1.36 \\
\hline$y_{11}$ & $-1.000-0.307 i$ & $0.282+1.166 i$ & $0.752+0.096 i$ & $-0.674+0.520 i$ \\
\hline$y_{13}$ & $-0.451+0.631 i$ & $0.031-0.880 i$ & $0.919-0.432 i$ & $1.026-0.542 i$ \\
\hline$y_{22}$ & $0.860+0.353 i$ & $0.097-1.088 i$ & $-0.905-0.194 i$ & $0.974+0.431 i$ \\
\hline$y_{31}$ & $0.667+0.397 i$ & $0.001-0.881 i$ & $0.941+0.383 i$ & $-1.070+0.450 i$ \\
\hline$y_{33}$ & $0.792-0.683 i$ & $-0.324+1.154 i$ & $0.746-0.136 i$ & $0.829-0.191 i$ \\
\hline
\end{tabular}

Table 3. Benchmark points for the neutrino sector, featuring both NH and IH in Cases I and II.

\begin{tabular}{|c|c|c|c|c|}
\hline & NH-I & NH-II & IH-I & IH-II \\
\hline$m_{1}[\mathrm{eV}]$ & $1.80 \times 10^{-3}$ & $2.59 \times 10^{-3}$ & $4.88 \times 10^{-2}$ & $4.89 \times 10^{-2}$ \\
\hline$m_{2}[\mathrm{eV}]$ & $8.90 \times 10^{-3}$ & $9.10 \times 10^{-3}$ & $4.96 \times 10^{-2}$ & $4.97 \times 10^{-2}$ \\
\hline$m_{3}[\mathrm{eV}]$ & $4.98 \times 10^{-2}$ & $4.99 \times 10^{-2}$ & $2.41 \times 10^{-3}$ & $3.50 \times 10^{-3}$ \\
\hline$\Delta m_{21}^{2}\left[10^{-5} \mathrm{eV}^{2}\right]$ & 7.60 & 7.60 & 7.50 & 7.48 \\
\hline$\left|\Delta m_{31}^{2}\right|\left[10^{-3} \mathrm{eV}^{2}\right]$ & 2.48 & 2.48 & 2.38 & 2.38 \\
\hline $\sin ^{2} \theta_{12} / 10^{-1}$ & 3.41 & 3.41 & 3.41 & 3.41 \\
\hline $\sin ^{2} \theta_{23} / 10^{-1}$ & 5.67 & 5.67 & 5.73 & 5.73 \\
\hline $\sin ^{2} \theta_{13} / 10^{-2}$ & 2.26 & 2.26 & 2.29 & 2.29 \\
\hline$J_{\mathrm{CP}} / 10^{-2}$ & -2.71 & -2.71 & -2.58 & -2.57 \\
\hline
\end{tabular}

Table 4. Neutrino masses and oscillation parameters associated to the four chosen benchmark points.

The neutrino masses, splittings and mixing angles associated to each BP are displayed in table 4. All the obtained neutrino oscillation parameters are consistent with the global fit in ref. [35]. In particular, the reproduced atmospheric and reactor angles lie comfortably in their respective $1 \sigma$ region, whereas the solar angle values are contained in the $2 \sigma$ range, very close to the $1 \sigma$ boundary.

\section{Quark sector}

The quark transformation properties under the family group $\Delta(27) \otimes Z_{4} \otimes Z_{4}^{\prime}$ are given in table 5. At leading order, the most general invariant Yukawa interactions can be written as

$$
\begin{gathered}
\mathcal{L}_{Y}^{q}=\frac{\sqrt{G}}{\left(\Lambda^{\prime}\right)^{\frac{5}{2}}}\left\{y_{u u} \sigma_{2}^{*} \bar{\Psi}_{U} \widetilde{H} \Psi_{u}+y_{c t} \sigma_{1}^{*} \bar{\Psi}_{C} \widetilde{H} \Psi_{t}+y_{t c} \sigma_{2}^{*} \bar{\Psi}_{T} \widetilde{H} \Psi_{c}+y_{t t} \sigma_{1} \bar{\Psi}_{T} \widetilde{H} \Psi_{t}\right. \\
+y_{d s} \sigma_{1}^{*} \bar{\Psi}_{U} H \Psi_{s}+y_{d b} \sigma_{1} \bar{\Psi}_{U} H \Psi_{b}+y_{s d} \sigma_{2}^{*} \bar{\Psi}_{C} H \Psi_{d}
\end{gathered}
$$




\begin{tabular}{|c|c|c|c|c|c|c|c|c|c|}
\hline Field & $\Psi_{U}$ & $\Psi_{C}$ & $\Psi_{T}$ & $\Psi_{u}$ & $\Psi_{c}$ & $\Psi_{t}$ & $\Psi_{d}$ & $\Psi_{s}$ & $\Psi_{b}$ \\
\hline$\Delta(27)$ & $\mathbf{1}_{0,2}$ & $\mathbf{1}_{0,1}$ & $\mathbf{1}_{0,0}$ & $\mathbf{1}_{0,2}$ & $\mathbf{1}_{0,0}$ & $\mathbf{1}_{0,2}$ & $\mathbf{1}_{0,1}$ & $\mathbf{1}_{0,0}$ & $\mathbf{1}_{0,1}$ \\
\hline$Z_{4}$ & $-i$ & $-i$ & $-i$ & 1 & 1 & $-i$ & 1 & $-i$ & $-i$ \\
\hline$Z_{4}^{\prime}$ & 1 & 1 & 1 & -1 & -1 & -1 & -1 & -1 & -1 \\
\hline
\end{tabular}

Table 5. Particle content and transformation properties of the quark sector under the flavor symmetry $\Delta(27) \otimes Z_{4} \otimes Z_{4}^{\prime}$.

$$
\left.+y_{s s} \sigma_{1} \bar{\Psi}_{C} H \Psi_{s}+y_{b b} \sigma_{1}^{*} \bar{\Psi}_{T} H \Psi_{b}\right\} \delta(y-L)+\text { h.c. }
$$

Again, after spontaneous electroweak and flavor symmetry breaking, the mass matrices for the up and down quark sectors read

$$
\begin{gathered}
m^{u}=\frac{1}{\left(L \Lambda^{\prime}\right)^{\frac{3}{2}}} \frac{v_{H}}{\sqrt{2}}\left(\begin{array}{ccc}
\widetilde{y}_{u u} v_{\sigma_{2}}^{*} / \Lambda^{\prime} & 0 & 0 \\
0 & 0 & \widetilde{y}_{c t} v_{\sigma_{1}}^{*} / \Lambda^{\prime} \\
0 & \widetilde{y}_{t c} v_{\sigma_{2}}^{*} / \Lambda^{\prime} & \widetilde{y}_{t t} v_{\sigma_{1}} / \Lambda^{\prime}
\end{array}\right), \\
m^{d}=\frac{1}{\left(L \Lambda^{\prime}\right)^{\frac{3}{2}}} \frac{v_{H}}{\sqrt{2}}\left(\begin{array}{ccc}
0 & \widetilde{y}_{d s} v_{\sigma_{1}}^{*} / \Lambda^{\prime} & \widetilde{y}_{d b} v_{\sigma_{1}} / \Lambda^{\prime} \\
\widetilde{y}_{s d} v_{\sigma_{2}}^{*} / \Lambda^{\prime} & \widetilde{y}_{s s} v_{\sigma_{1}} / \Lambda^{\prime} & 0 \\
0 & 0 & \widetilde{y}_{b b} v_{\sigma_{1}}^{*} / \Lambda^{\prime}
\end{array}\right) .
\end{gathered}
$$

where

$$
\begin{aligned}
& \widetilde{y}_{u_{i} u_{j}}=y_{u_{i} u_{j}} F\left(L, c_{Q_{i}}, c_{u_{j}}\right), \\
& \widetilde{y}_{d_{i} d_{j}}=y_{d_{i} d_{j}} F\left(L, c_{Q_{i}}, c_{d_{j}}\right) .
\end{aligned}
$$

The up-type quark mass matrix is already block-diagonal. The diagonalization of the down-type mass matrix $m^{d}$ requires a more careful treatment. For the sake of simplicity, in the following analysis we denote the $i j$ element of $m^{u}\left(m^{d}\right)$ as $m_{i j}^{u}\left(m_{i j}^{d}\right)$. The product of the down-type mass matrix and its adjoint

$$
m^{d} m^{d \dagger}=\left(\begin{array}{ccc}
\left|m_{12}^{d}\right|^{2}+\left|m_{13}^{d}\right|^{2} & m_{12}^{d} m_{22}^{d *} & m_{13}^{d} m_{33}^{d *} \\
m_{12}^{d *} m_{22}^{d} & \left|m_{21}^{d}\right|^{2}+\left|m_{22}^{d}\right|^{2} & 0 \\
m_{13}^{d *} m_{33}^{d} & 0 & \left|m_{33}^{d}\right|^{2}
\end{array}\right)
$$

can be diagonalized in two steps: in first place, an approximate block diagonalization

$$
U^{d \prime \dagger} m^{d} m^{d \dagger} U^{d \prime} \simeq\left(\begin{array}{ccc}
\left|m_{12}^{d}\right|^{2} & m_{12}^{d} m_{22}^{d *} & 0 \\
m_{12}^{d *} m_{22}^{d} & \left|m_{21}^{d}\right|^{2}+\left|m_{22}^{d}\right|^{2} & 0 \\
0 & 0 & \left|m_{33}^{d}\right|^{2}
\end{array}\right),
$$

is accomplished with the aid of the transformation matrix

$$
U^{d \prime} \simeq\left(\begin{array}{ccc}
1 & 0 & \epsilon \\
0 & 1 & 0 \\
-\epsilon^{*} & 0 & 1
\end{array}\right)
$$


and subsequently the diagonalization is completed through a unitary rotation of the upper block. This approximation is consistent provided $\left|m_{33}^{d}\right| \gg\left|m_{12}^{d}\right|,\left|m_{13}^{d}\right|,\left|m_{22}^{d}\right|$ and $|\epsilon| \ll 1$. The resulting diagonalization matrices for the up and down sectors can be parameterized as

$$
\begin{aligned}
& U_{u}=\left(\begin{array}{ccc}
1 & 0 & 0 \\
0 & \cos \theta_{u} & \sin \theta_{u} e^{i \varphi_{u}} \\
0-\sin \theta_{u} e^{-i \varphi_{u}} & \cos \theta_{u}
\end{array}\right), \\
& U_{d} \simeq\left(\begin{array}{ccc}
\cos \theta_{d} & \sin \theta_{d} e^{i \varphi_{d}} & \epsilon \\
-\sin \theta_{d} e^{-i \varphi_{d}} & \cos \theta_{d} & 0 \\
-\epsilon^{*} \cos \theta_{d} & -\epsilon^{*} \sin \theta_{d} e^{i \varphi_{d}} & 1
\end{array}\right),
\end{aligned}
$$

with

$$
\begin{aligned}
& \tan 2 \theta_{u}=2\left|Z_{u}\right| / X_{u}^{-}, \quad \varphi_{u}=\arg Z_{u}, \\
& \tan 2 \theta_{d}=2\left|Z_{d}\right| / X_{d}^{-}, \quad \varphi_{d}=\arg Z_{d}, \quad \epsilon=B_{d} / A_{d},
\end{aligned}
$$

and

$$
\begin{array}{rll}
X_{u}^{ \pm}=\left|m_{33}^{u}\right|^{2}+\left|m_{32}^{u}\right|^{2} \pm\left|m_{23}^{u}\right|^{2}, & Y_{u}=m_{23}^{u} m_{32}^{u *}, & Z_{u}=m_{23}^{u} m_{33}^{u *}, \\
X_{d}^{ \pm}=\left|m_{22}^{d}\right|^{2}+\left|m_{21}^{d}\right|^{2} \pm\left|m_{12}^{d}\right|^{2}, & Y_{d}=m_{12}^{d} m_{21}^{d *}, & Z_{d}=m_{12}^{d} m_{22}^{d *}, \\
A_{d} & =\left|m_{33}^{d}\right|^{2}-\left|m_{12}^{d}\right|^{2}-\left|m_{13}^{d}\right|^{2}, & B_{d}=m_{13}^{d} m_{33}^{d *} .
\end{array}
$$

Correspondingly, the quark mass eigenvalues can be expressed in terms of $M^{ \pm}$, defined in eq. (3.19), as

$$
\begin{aligned}
& m_{u}=\left|m_{11}^{u}\right|, \quad m_{c}=\frac{1}{\sqrt{2}} M^{-}\left(X_{u}^{+}, Y_{u}\right), \quad m_{t}=\frac{1}{\sqrt{2}} M^{+}\left(X_{u}^{+}, Y_{u}\right), \\
& m_{d}=\frac{1}{\sqrt{2}} M^{-}\left(X_{d}^{+}, Y_{d}\right), \quad m_{s}=\frac{1}{\sqrt{2}} M^{+}\left(X_{d}^{+}, Y_{d}\right), \quad m_{b}=\left|m_{33}^{b}\right|,
\end{aligned}
$$

so that the CKM matrix is given by

$$
\begin{aligned}
& V_{\mathrm{CKM}}=U_{u}^{\dagger} U_{d} \\
& \simeq\left(\begin{array}{ccc}
\cos \theta_{d} & e^{i \varphi_{d}} \sin \theta_{d} & \epsilon \\
-e^{-i \varphi_{d}} \cos \theta_{u} \sin \theta_{d}-e^{i \varphi_{u}} \sin \theta_{u} \cos \theta_{d} \epsilon^{*} & \cos \theta_{d} \cos \theta_{u}-e^{i\left(\varphi_{u}+\varphi_{d}\right)} \sin \theta_{u} \sin \theta_{d} \epsilon^{*} & -e^{i \varphi_{u}} \sin \theta_{u} \\
-e^{-i\left(\varphi_{d}+\varphi_{u}\right)} \sin \theta_{d} \sin \theta_{u}-\cos \theta_{u} \cos \theta_{d} \epsilon^{*} & e^{-i \varphi_{u}} \cos \theta_{d} \sin \theta_{u}-e^{i \varphi_{d}} \cos \theta_{u} \sin \theta_{d} \epsilon^{*} & \cos \theta_{u}
\end{array}\right) .
\end{aligned}
$$

Hence, the quark sector Dirac CP phase (in PDG convention) and the Jarlskog invariant take the form

$$
\begin{aligned}
\delta_{\mathrm{CP}}^{q} & =\pi-\arg (\epsilon)+\varphi_{d}+\varphi_{u}, \\
J_{\mathrm{CP}}^{q} & \simeq \frac{1}{4}|\epsilon| \sin 2 \theta_{d} \sin 2 \theta_{u} \sin \delta_{\mathrm{CP}}^{q} .
\end{aligned}
$$

According to eq. (4.3), the size of up and down mass matrix elements is determined by the overlap of the 5D quark field zero mode profiles, i.e., $m_{i j}^{u} \propto f_{L}^{(0)}\left(L, c_{Q_{i}}\right) f_{R}^{(0)}\left(L, c_{u_{j}}\right)$ and $m_{i j}^{d} \propto f_{L}^{(0)}\left(L, c_{Q_{i}}\right) f_{R}^{(0)}\left(L, c_{d_{j}}\right)$. If the wave function localization parameters $c_{Q_{i}}, c_{u_{i}}, c_{d_{i}}$ are chosen such that the quark zero mode profiles obey

$$
f_{L}^{(0)}\left(L, c_{U}\right) \ll f_{L}^{(0)}\left(L, c_{C}\right) \ll f_{L}^{(0)}\left(L, c_{T}\right),
$$




$$
\begin{aligned}
& f_{R}^{(0)}\left(L, c_{u}\right) \ll f_{R}^{(0)}\left(L, c_{c}\right) \ll f_{R}^{(0)}\left(L, c_{t}\right), \\
& f_{R}^{(0)}\left(L, c_{d}\right) \ll f_{R}^{(0)}\left(L, c_{s}\right) \ll f_{R}^{(0)}\left(L, c_{b}\right),
\end{aligned}
$$

then the elements of $m^{u}$ and $m^{d}$ approximately satisfy

$$
m_{11}^{u} \ll m_{23}^{u} \sim m_{32}^{u} \ll m_{33}^{u}, \quad m_{12}^{d} \sim m_{21}^{d} \ll m_{22}^{d} \ll m_{33}^{d}, \quad m_{13}^{d} \ll m_{33}^{d},
$$

justifying the perturbative diagonalization performed on $m^{d} m^{d \dagger}$. These relations imply that $X_{u, d}^{+} \gg\left|Y_{u, d}\right|$ holds, and therefore, a rough estimate for the mixing parameters and quark mass spectrum is

$$
\begin{array}{rlrlrl}
\theta_{u} & \sim\left|\frac{m_{23}^{u}}{m_{33}^{u}}\right| \sim \frac{f_{L}^{(0)}\left(L, c_{C}\right)}{f_{L}^{(0)}\left(L, c_{T}\right)}, & \theta_{d} & \sim\left|\frac{m_{12}^{d}}{m_{22}^{d}}\right| \sim \frac{f_{L}^{(0)}\left(L, c_{U}\right)}{f_{L}^{(0)}\left(L, c_{C}\right)}, & |\epsilon| \sim\left|\frac{m_{13}^{d}}{m_{33}^{d}}\right| \sim \frac{f_{L}^{(0)}\left(L, c_{U}\right)}{f_{L}^{(0)}\left(L, c_{T}\right)}, \\
m_{u} & \sim\left|m_{11}^{u}\right|, & m_{c} \sim\left|\frac{m_{23}^{u} m_{32}^{u}}{m_{33}^{u}}\right|, & m_{t} \sim\left|m_{33}^{u}\right|, \\
m_{d} \sim\left|\frac{m_{12}^{d} m_{21}^{d}}{m_{22}^{d}}\right|, & m_{s} \sim\left|m_{22}^{d}\right|, & m_{b} \sim\left|m_{33}^{d}\right| .
\end{array}
$$

Thus, in order to reproduce plausible quark masses and mixings, namely:

$$
\begin{aligned}
\theta_{u} \sim 10^{-1}, \quad \theta_{d} & \sim 10^{-2}, \quad|\epsilon| \sim 10^{-3}, \\
m_{u}: m_{c}: m_{t} & \sim 10^{-5}: 10^{-2}: 1, \\
m_{d}: m_{s}: m_{b} & \sim 10^{-3}: 10^{-2}: 1,
\end{aligned}
$$

the quark zero mode profiles must observe the following hierarchy:

$$
\begin{gathered}
f_{L}^{(0)}\left(L, c_{U}\right): f_{L}^{(0)}\left(L, c_{C}\right): f_{L}^{(0)}\left(L, c_{T}\right) \sim 10^{-3}: 10^{-1}: 1, \\
f_{R}^{(0)}\left(L, c_{u}\right): f_{R}^{(0)}\left(L, c_{c}\right): f_{R}^{(0)}\left(L, c_{t}\right) \sim 10^{-2}: 10^{-1}: 1, \\
f_{R}^{(0)}\left(L, c_{d}\right): f_{R}^{(0)}\left(L, c_{s}\right): f_{R}^{(0)}\left(L, c_{b}\right) \sim 10^{-1}: 10^{-1}: 1 .
\end{gathered}
$$

To conclude this section, an explicit realization of quark masses and mixings is presented. The choice $c_{U}=1.97, c_{C}=1.92, c_{T}=1.83, c_{u}=-0.76, c_{c}=-0.62, c_{t}=-0.56$, $c_{d}=-0.74, c_{s}=-0.69, c_{b}=-0.68, y_{u u}=-0.438-0.954 i, y_{c t}=-0.360-1.038 i$, $y_{t c}=1.147-0.273 i, y_{t t}=-0.372-1.073 i, y_{d s}=-0.966-0.285 i, y_{d b}=0.290+0.400 i$, $y_{s d}=0.838-0.226 i, y_{s s}=-0.703-0.207 i, y_{b b}=0.637-0.879 i$, generates the quark mass spectrum

$$
\begin{array}{lll}
m_{u}=2.30 \mathrm{MeV}, & m_{c}=1.275 \mathrm{GeV}, & m_{t}=173 \mathrm{GeV}, \\
m_{d}=4.80 \mathrm{MeV}, & m_{s}=95.0 \mathrm{MeV}, & m_{b}=4.18 \mathrm{GeV},
\end{array}
$$

and fixes the magnitude of $V_{C K M}$ elements at

$$
\left|V_{\mathrm{CKM}}\right|=\left(\begin{array}{ccc}
0.974 & 0.225 & 0.0035 \\
0.225 & 0.973 & 0.0414 \\
0.0089 & 0.041 & 0.999
\end{array}\right) .
$$


Finally, the obtained values for the Dirac CP phase and the Jarlskog invariant are

$$
\delta_{\mathrm{CP}}^{q}=1.25, \quad J_{\mathrm{CP}}^{q}=3.06 \times 10^{-5} .
$$

The resulting quark masses and mixings are consistent with the current experimental data [1], and the precision of the results can be improved by incorporating high order corrections, addressed in the next section.

\section{$5 \quad$ High order corrections}

From the particle content and above transformation properties, one finds that nontrivial high order corrections to the charged lepton sector are absent in the present model. The next-to-leading order (NLO) corrections to the neutrino Yukawa interactions are given by

$$
\delta \mathcal{L}_{Y}^{\nu}=\sqrt{G} \frac{x_{2}}{\left(\Lambda^{\prime}\right)^{\frac{9}{2}}}\left[\left(\xi^{*} \xi^{*}\right)_{\mathbf{3}} \sigma_{2}^{*} \bar{\Psi}_{l}\right]_{\mathbf{1}_{0,0}} \widetilde{H} \Psi_{\nu_{2}} \delta(y-L)+\text { h.c. }
$$

However, the contribution of these terms to the neutrino masses and mixing parameters can be absorbed by a proper redefinition of the parameter $y_{22}$ after SSB. Hence, in order to estimate the effects of higher order corrections in this sector, we need to investigate the Yukawa terms involving an additional $\left(v_{\mathrm{IR}} / \Lambda^{\prime}\right)^{2}$ suppression with respect to the lowest order terms in eq. (3.1), where we have introduced $v_{\text {IR }}$ to characterize the magnitude of $v_{\xi} \sim v_{\sigma_{1}} \sim v_{\sigma_{2}}$.

The contraction of the field products $\bar{\Psi}_{l} \widetilde{H} \Psi_{\nu_{1}}, \bar{\Psi}_{l} \widetilde{H} \Psi_{\nu_{3}}$, transforming as $(\overline{\mathbf{3}},-1,-1)$ under $\Delta(27) \otimes Z_{4} \otimes Z_{4}^{\prime}$, with the flavon operators

$$
\frac{1}{\left(\Lambda^{\prime}\right)^{\frac{11}{2}}}\left(\xi \xi^{*}\right)_{\mathbf{1}_{a, 2}} \xi \sigma_{1}, \quad \frac{1}{\left(\Lambda^{\prime}\right)^{\frac{11}{2}}}\left(\xi \xi^{*}\right)_{\mathbf{1}_{a, 1}} \xi \sigma_{1}^{*}, \quad \frac{1}{\left(\Lambda^{\prime}\right)^{\frac{11}{2}}} \xi \sigma_{1}^{3}, \quad \frac{1}{\left(\Lambda^{\prime}\right)^{\frac{11}{2}}} \xi \sigma_{1}^{* 3}
$$

as well as the combination of $\bar{\Psi}_{l} \widetilde{H} \Psi_{\nu_{2}} \sim(\overline{\mathbf{3}}, i,-1)$ and

$$
\frac{1}{\left(\Lambda^{\prime}\right)^{\frac{11}{2}}}\left(\xi \xi^{*}\right)_{\mathbf{1}_{a, b}} \xi \sigma_{2}, \quad \frac{1}{\left(\Lambda^{\prime}\right)^{\frac{11}{2}}} \xi \sigma_{1}^{2} \sigma_{2}, \quad \frac{1}{\left(\Lambda^{\prime}\right)^{\frac{11}{2}}} \xi \sigma_{1}^{* 2} \sigma_{2},
$$

provide the desired high order corrections to the neutrino Yukawa interactions. In the above expressions, the indices $a, b=0,1,2$ label the different singlets of $\Delta(27)$. Additional terms that can be absorbed into $y_{11}, y_{13}, y_{22}, y_{31}$ and $y_{33}$ have been omitted. Taking into consideration these corrections, the neutrino mass matrix $m_{\nu}$ can be roughly written as

$$
m_{\nu} \simeq \frac{1}{\left(L \Lambda^{\prime}\right)^{\frac{3}{2}}} \frac{v_{H}}{\sqrt{2}}\left[\frac{v_{\xi}}{\Lambda^{\prime}}\left(\begin{array}{ccc}
\widetilde{y}_{11} \frac{v_{\sigma_{1}}}{\Lambda^{\prime}} & 0 & \widetilde{y}_{13} \frac{v_{\sigma_{1}}}{\Lambda^{\prime}} \\
0 & \widetilde{y}_{22} \frac{v_{\sigma_{2}}}{\Lambda^{\prime}} & 0 \\
\widetilde{y}_{31} \frac{v_{\sigma_{1}}^{*}}{\Lambda^{\prime}} & 0 & \widetilde{y}_{33} \frac{v_{\sigma_{1}}^{*}}{\Lambda^{\prime}}
\end{array}\right)+\left(\frac{v_{\mathrm{IR}}}{\Lambda^{\prime}}\right)^{4}\left(\begin{array}{ccc}
0 & \widetilde{x}_{12} & 0 \\
\widetilde{x}_{21} & 0 & \widetilde{x}_{23} \\
0 & \widetilde{x}_{32} & 0
\end{array}\right)\right]
$$

with $\widetilde{x}_{i j}=x_{i j} F\left(L, c_{l}, c_{\nu_{j}}\right)$, and $x_{i j}$ as dimensionless parameters of order $O(1)$.

Working under the same numerical framework established in section 3, one can readily estimate the shift in the neutrino oscillation parameters induced by high order corrections of the Yukawa interaction. Particularly, in Case I, taking $x_{i j}$ as random complex numbers 
with magnitudes ranging from 2 to 6 , and $v_{\mathrm{IR}}=0.1$, the resulting deviations in the neutrino mixing parameters with respect to their LO values can be estimated as

$$
\delta s_{12}^{2} \sim 0.01 \quad \delta s_{23}^{2} \sim 0.01 \quad \delta s_{13}^{2} \sim 0.001 \quad \delta J_{C P} \sim 0.001 .
$$

On the other hand, the corrections to the neutrino mass splittings are negligible

$$
\delta\left(\Delta m_{21}^{2}\right) \sim 10^{-7} \mathrm{eV}^{2}, \quad \delta\left(\Delta\left|m_{31}^{2}\right|\right) \sim 10^{-6} \mathrm{eV}^{2} .
$$

From eq. (5.5), it is clear that high order corrections can easily drive $s_{12}^{2}$ into its $1 \sigma$ region while keeping the remaining parameters optimal.

Turning to the quark sector, every bilinear formed by $\bar{\Psi}_{Q_{i}}$ and $\Psi_{u_{i}}$ or $\Psi_{d_{i}}$ can produce a high order correction to the Yukawa interaction whenever it is contracted with the adequate cubic flavon operator. Beside terms that can be absorbed by a redefinition of $y_{u_{i} u_{j}}$ or $y_{d_{i} d_{j}}$ in eq. (4.1), all the NLO contributions can be classified into three categories:

- Invariant products of $\bar{\Psi}_{U} \widetilde{H} \Psi_{c}, \bar{\Psi}_{C} \widetilde{H} \Psi_{u}, \bar{\Psi}_{T} H \Psi_{d} \sim\left(\mathbf{1}_{0,1}, i,-1\right)$ with

$$
\frac{1}{\left(\Lambda^{\prime}\right)^{\frac{9}{2}}}\left(\xi \xi^{*}\right)_{\mathbf{1}_{0,2}} \sigma_{2}^{*}, \quad \quad \frac{1}{\left(\Lambda^{\prime}\right)^{\frac{9}{2}}} \sigma_{1}^{2} \sigma_{2}^{*} \text {. }
$$

- Invariant products of $\bar{\Psi}_{U} \widetilde{H} \Psi_{t}, \bar{\Psi}_{C} H \Psi_{b}, \bar{\Psi}_{T} H \Psi_{s} \sim\left(\mathbf{1}_{0,0}, 1,-1\right)$ with

$$
\frac{1}{\left(\Lambda^{\prime}\right)^{\frac{9}{2}}}\left(\xi \xi^{*}\right)_{\mathbf{1}_{0,2}} \sigma_{1}, \quad \frac{1}{\left(\Lambda^{\prime}\right)^{\frac{9}{2}}}\left(\xi \xi^{*}\right)_{\mathbf{1}_{0,1}} \sigma_{1}^{*}, \quad \frac{1}{\left(\Lambda^{\prime}\right)^{\frac{9}{2}}} \sigma_{1}^{3}, \quad \frac{1}{\left(\Lambda^{\prime}\right)^{\frac{9}{2}}} \sigma_{1}^{* 3}
$$

- Invariant products of $\bar{\Psi}_{C} \widetilde{H} \Psi_{c}, \bar{\Psi}_{T} \widetilde{H} \Psi_{u}$ and $\bar{\Psi}_{U} H \Psi_{d} \sim\left(\mathbf{1}_{0,2}, i,-1\right)$ with

$$
\frac{1}{\left(\Lambda^{\prime}\right)^{\frac{9}{2}}}\left(\xi \xi^{*}\right)_{\mathbf{1}_{0,1}} \sigma_{2}^{*}, \quad \quad \frac{1}{\left(\Lambda^{\prime}\right)^{\frac{9}{2}}} \sigma_{1}^{* 2} \sigma_{2}^{*}
$$

Again, after symmetry breaking, the quark mass matrices $m_{u}$ and $m_{d}$ can be approximately written as

$$
\begin{aligned}
& m^{u}=\frac{1}{\left(L \Lambda^{\prime}\right)^{\frac{3}{2}}} \frac{v_{H}}{\sqrt{2}}\left[\left(\begin{array}{ccc}
\widetilde{y}_{u u} v_{\sigma_{2}}^{*} / \Lambda^{\prime} & 0 & 0 \\
0 & 0 & \widetilde{y}_{c t} v_{\sigma_{1}}^{*} / \Lambda^{\prime} \\
0 & \widetilde{y}_{t c} v_{\sigma_{2}}^{*} / \Lambda^{\prime} & \widetilde{y}_{t t} v_{\sigma_{1}} / \Lambda^{\prime}
\end{array}\right)+\left(\frac{v_{\mathrm{IR}}}{\Lambda^{\prime}}\right)^{3}\left(\begin{array}{ccc}
0 & \widetilde{x}_{u c} & \widetilde{x}_{u t} \\
\widetilde{x}_{c u} & \widetilde{x}_{c c} & 0 \\
\widetilde{x}_{t u} & 0 & 0
\end{array}\right)\right], \\
& m^{d}=\frac{1}{\left(L \Lambda^{\prime}\right)^{\frac{3}{2}}} \frac{v_{H}}{\sqrt{2}}\left[\left(\begin{array}{ccc}
0 & \widetilde{y}_{d s} v_{\sigma_{1}}^{*} / \Lambda^{\prime} & \widetilde{y}_{d b} v_{\sigma_{1}} / \Lambda^{\prime} \\
\widetilde{y}_{s d} v_{\sigma_{2}}^{*} / \Lambda^{\prime} & \widetilde{y}_{s s} v_{\sigma_{1}} / \Lambda^{\prime} & 0 \\
0 & 0 & \widetilde{y}_{b b} v_{\sigma_{1}} / \Lambda^{\prime}
\end{array}\right)+\left(\frac{v_{\mathrm{IR}}}{\Lambda^{\prime}}\right)^{3}\left(\begin{array}{ccc}
\widetilde{x}_{d d} & 0 & 0 \\
0 & 0 & \widetilde{x}_{s b} \\
\widetilde{x}_{b d} & \widetilde{x}_{b s} & 0
\end{array}\right)\right] .
\end{aligned}
$$

Here we have defined $\widetilde{x}_{u_{i} u_{j}}=x_{u_{i} u_{j}} F\left(L, c_{Q_{i}}, c_{u_{j}}\right)$ and $\widetilde{x}_{d_{i} d_{j}}=x_{d_{i} d_{j}} F\left(L, c_{Q_{i}}, c_{d_{j}}\right)$, where the couplings $x_{u_{i} u_{j}}$ and $x_{d_{i} d_{j}}$ represent dimensionless parameters of order $O(1)$. As a numerical example, taking $x_{u_{i} u_{j}}, x_{d_{i} d_{j}}$ as random complex numbers with magnitudes ranging from 1 to 4 for $x_{u c}, x_{c u}, x_{b d}, x_{c c}, x_{t u}, x_{d d}$, and from 2 to 6 for $x_{u t}, x_{s b}, x_{b s}$, while keeping the 


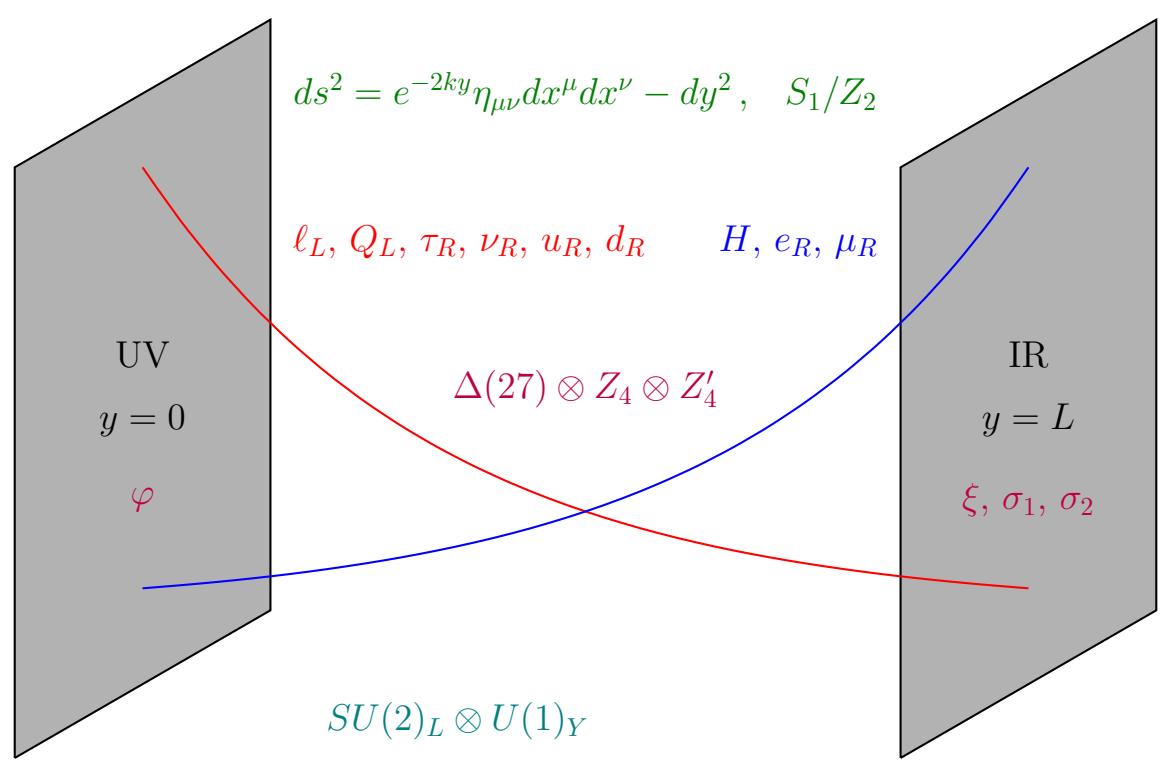

Figure 2. Pictorial description of the basic warped model structure, showing the UV (IR) peaked nature of the standard model fields.

values of $c_{Q_{i}}, c_{u_{i}}, c_{d_{i}}, y_{u_{i} u_{j}}$ and $y_{d_{i} d_{j}}$ reported in section 4 , the order of deviation with respect to the $\mathrm{LO}$ values of the quark masses is

$$
\begin{array}{rlrl}
\delta m_{u} & \sim 0.001 \mathrm{MeV}, & \delta m_{c} \sim 10 \mathrm{MeV}, & \delta m_{t} \sim 0.1 \mathrm{MeV}, \\
\delta m_{d} \sim 0.1 \mathrm{MeV}, & \delta m_{s} \sim 0.1 \mathrm{MeV}, & \delta m_{b} \sim 0.5 \mathrm{MeV} .
\end{array}
$$

The corresponding correction to the first order CKM matrix is of order

$$
\delta\left|V_{\mathrm{CKM}}\right| \sim\left(\begin{array}{ccc}
0.001 & 0.005 & 0.0001 \\
0.005 & 0.001 & 0.001 \\
0.0005 & 0.001 & 0.00005
\end{array}\right),
$$

and the values for the quark $\mathrm{CP}$ violating phase and the Jarlskog invariant are displaced by

$$
\delta\left(\delta_{\mathrm{CP}}^{q}\right) \sim 0.1, \quad \delta J_{\mathrm{CP}}^{q} \sim 10^{-6} .
$$

As for the lepton sector, it is not difficult to find parameter values reproducing the quark mass and mixing parameters required to fit the current experimentally observed values.

\section{Conclusions}

We have proposed a five-dimensional warped model in which all standard model fields propagate into the bulk. Its structure is summarized in the "cartoon" depicted in figure 2. Mass hierarchies in principle arise from an adequate choice of the bulk shape parameters, while fermion mixing angles are constrained by relations which follow from the postulated $\Delta(27)$ flavor symmetry group, broken on the branes by a set of flavon fields. The neutrino 
mixing parameters and the Dirac CP violation phase are described in terms of just two independent parameters at leading order. This leads to stringent predictions for the lepton mixing matrix which should be tested in future neutrino oscillation experiments. Likewise the scheme also includes the quark sector, providing an adequate description of the quark mixing matrix. The effect of next-to-leading order contributions is estimated to be fully consistent with the experimental requirements.

\section{Acknowledgments}

This work is supported by the National Natural Science Foundation of China under Grant Nos. 11275188, 11179007 and 11522546; by the Spanish grants FPA2014-58183-P, Multidark CSD2009-00064, SEV-2014-0398 (MINECO) and PROMETEOII/2014/084 (Generalitat Valenciana). A.D.R. and C.A.V-A. acknowledge support from CONACyT (Mexico), grants 250610 and 251357.

\section{A The profile of the Higgs zero mode}

In this appendix, we shall show that the Higgs zero mode profile in eq. (2.4) can be achieved for a proper choice of boundary conditions. Following the original idea proposed in the appendix A of ref. [48], the action of the Higgs field reads as:

$$
S_{H}^{\text {bulk }}=\int d^{5} x \sqrt{G}\left\{G^{M N} \operatorname{Tr}\left[\left(D_{M} H\right)^{\dagger} D_{N} H\right]-m_{H}^{2} \operatorname{Tr}\left(H^{\dagger} H\right)-V_{\mathrm{UV}}(H) \delta(y)-V_{\mathrm{IR}}(H) \delta(y-L)\right\} .
$$

In order to generate a VEV close to the IR brane, the potential $V_{\mathrm{IR}}(H)$ is assumed to have the usual "mexican hat" form [48]

$$
V_{\mathrm{IR}}(H)=\frac{\lambda}{2}\left[\operatorname{Tr}\left(H^{\dagger} H\right)-\frac{v_{\mathrm{TeV}}^{2}}{2}\right]^{2}
$$

On the UV brane, only a mass term is added [42, 47, 48]

$$
V_{\mathrm{UV}}(H)=-m_{\mathrm{UV}} \operatorname{Tr}\left(H^{\dagger} H\right)
$$

The variation of the action yields both the equations of motion as well as boundary conditions for the bulk Higgs field as follows:

$$
\begin{aligned}
\left(e^{2 k y} \partial_{y} e^{-4 k y} \partial_{y}-\partial^{\mu} \partial_{\mu}-e^{-2 k y} m_{H}^{2}\right) H & =0, \\
\partial_{y} H+\left.\lambda H\left[\operatorname{Tr}\left(H^{\dagger} H\right)-v_{\mathrm{TeV}}^{2} / 2\right]\right|_{y=L} & =0, \\
\partial_{y} H-\left.m_{\mathrm{UV}} H\right|_{y=0} & =0 .
\end{aligned}
$$

The Higgs potential in eq. (A.1) leads to non-zero VEV of the Higgs doublet $H$ with

$$
\langle H\rangle=\frac{1}{\sqrt{2}}\left(\begin{array}{c}
0 \\
v(y)
\end{array}\right)=\frac{1}{\sqrt{2}}\left(\begin{array}{c}
0 \\
v_{H}
\end{array}\right) \frac{f_{H}(y)}{\sqrt{L}},
$$


where $v(y) \equiv v_{H} f_{H}(y)$, and $v_{H}$ denotes the effective $4 \mathrm{D}$ VEV of the zero mode of $\phi_{0}$. Obviously $f_{H}(y)$ fulfills the following set of conditions:

$$
\begin{aligned}
\left(e^{2 k y} \partial_{y} e^{-4 k y} \partial_{y}-e^{-2 k y} m_{H}^{2}\right) f_{H}(y) & =0, \\
\partial_{y} f_{H}(y)+\left.\lambda f_{H}(y)\left[v_{H}^{2} f_{H}^{2}(y)-v_{\mathrm{TeV}}^{2} / 2\right]\right|_{y=L} & =0, \\
\partial_{y} f_{H}(y)-\left.m_{\mathrm{UV}} f_{H}(y)\right|_{y=0} & =0 .
\end{aligned}
$$

The most general solution of eq. (A.8) is given by

$$
f_{H}(y)=a e^{(2+\beta) k y}+b e^{(2-\beta) k y},
$$

where $\beta=\sqrt{4+m_{H}^{2} / k^{2}}, a$ and $b$ are determined by the boundary conditions in eq. (A.9) and (A.10). From eq. (A.10) we have

$$
\frac{a}{b}=-\frac{(2-\beta) k-m_{\mathrm{UV}}}{(2+\beta) k-m_{\mathrm{UV}}}
$$

In the case of $m_{\mathrm{UV}}=(2-\beta) k$, we have $a=0$ such that only the term $e^{(2-\beta) k y}$ is picked out. Considering further the normalization condition $\int_{0}^{L} e^{-2 k y} f_{H}^{2}(y)=1$, we obtain

$$
f_{H}(y)=\sqrt{\frac{2 k L(1-\beta)}{1-e^{-2(1-\beta) k L}}} e^{k L} e^{(2-\beta) k(y-L)} .
$$

It is exactly the desired Higgs profile zero mode. Notice that the boundary conditions of the Higgs field is of the "mixed" type, as shown in eqs. (A.5), (A.6), and that a Higgs zero mode can not be obtained in the absence of $V_{\mathrm{IR}}(H)$ and $V_{\mathrm{UV}}(H)$.

\section{B Group theory of $\Delta(27)$ and its representation}

The $\Delta(27)$ group is isomorphic to $\left(Z_{3} \otimes Z_{3}\right) \rtimes Z_{3}$. It can be conveniently expressed in terms of three generators $a, a^{\prime}$ and $b$ which satisfy the following relations:

$$
\begin{aligned}
a^{3} & =a^{\prime 3}=b^{3}=1, & a a^{\prime} & =a^{\prime} a, \\
b a b^{-1} & =a^{-1} a^{\prime-1}, & b a^{\prime} b^{-1} & =a .
\end{aligned}
$$

All $\Delta(27)$ elements can be written into the form $b^{k} a^{m} a^{\prime n}$, with $k, m, n=0,1,2$. The group has 11 conjugacy classes, given by

$$
\begin{aligned}
1 C_{1} & =\{1\}, \\
1 C_{1}^{(1)} & =\left\{a a^{\prime 2}\right\}, \\
1 C_{1}^{(2)} & =\left\{a^{2} a^{\prime}\right\}, \\
3 C_{3}^{(0,1)} & =\left\{a, a^{\prime}, a^{2} a^{\prime 2}\right\}, \\
3 C_{3}^{(0,2)} & =\left\{a^{2}, a^{\prime 2}, a a^{\prime}\right\}, \\
3 C_{3}^{(1,0)} & =\left\{b, b a a^{\prime 2}, b a^{2} a^{\prime}\right\},
\end{aligned}
$$




\begin{tabular}{|c|c|c|c|c|c|c|c|c|c|c|c|}
\hline & $\chi_{\mathbf{1}_{0,0}}$ & $\chi_{\mathbf{1}_{0,1}}$ & $\chi_{\mathbf{1}_{0,2}}$ & $\chi_{\mathbf{1}_{1,0}}$ & $\chi_{\mathbf{1}_{1,1}}$ & $\chi_{\mathbf{1}_{1,2}}$ & $\chi_{\mathbf{1}_{2,0}}$ & $\chi_{\mathbf{1}_{2,1}}$ & $\chi_{\mathbf{1}_{2,2}}$ & $\chi_{\mathbf{3}}$ & $\chi_{\overline{\mathbf{3}}}$ \\
\hline $1 C_{1}$ & 1 & 1 & 1 & 1 & 1 & 1 & 1 & 1 & 1 & 3 & 3 \\
\hline $1 C_{1}^{(1)}$ & 1 & 1 & 1 & 1 & 1 & 1 & 1 & 1 & 1 & $3 \omega^{2}$ & $3 \omega$ \\
\hline $1 C_{1}^{(2)}$ & 1 & 1 & 1 & 1 & 1 & 1 & 1 & 1 & 1 & $3 \omega$ & $3 \omega^{2}$ \\
\hline $3 C_{3}^{(0,1)}$ & 1 & $\omega$ & $\omega^{2}$ & 1 & $\omega$ & $\omega^{2}$ & 1 & $\omega$ & $\omega^{2}$ & 0 & 0 \\
\hline $3 C_{3}^{(0,2)}$ & 1 & $\omega^{2}$ & $\omega$ & 1 & $\omega^{2}$ & $\omega$ & 1 & $\omega^{2}$ & $\omega$ & 0 & 0 \\
\hline $3 C_{3}^{(1,0)}$ & 1 & 1 & 1 & $\omega$ & $\omega$ & $\omega$ & $\omega^{2}$ & $\omega^{2}$ & $\omega^{2}$ & 0 & 0 \\
\hline $3 C_{3}^{(1,1)}$ & 1 & $\omega$ & $\omega^{2}$ & $\omega$ & $\omega^{2}$ & 1 & $\omega^{2}$ & 1 & $\omega$ & 0 & 0 \\
\hline $3 C_{3}^{(1,2)}$ & 1 & $\omega^{2}$ & $\omega$ & $\omega$ & 1 & $\omega^{2}$ & $\omega^{2}$ & $\omega$ & 1 & 0 & 0 \\
\hline $3 C_{3}^{(2,0)}$ & 1 & 1 & 1 & $\omega^{2}$ & $\omega^{2}$ & $\omega^{2}$ & $\omega$ & $\omega$ & $\omega$ & 0 & 0 \\
\hline $3 C_{3}^{(2,1)}$ & 1 & $\omega$ & $\omega^{2}$ & $\omega^{2}$ & 1 & $\omega$ & $\omega$ & $\omega^{2}$ & 1 & 0 & 0 \\
\hline $3 C_{3}^{(2,2)}$ & 1 & $\omega^{2}$ & $\omega$ & $\omega^{2}$ & $\omega$ & 1 & $\omega$ & 1 & $\omega^{2}$ & 0 & 0 \\
\hline
\end{tabular}

Table 6. Character table of $\Delta(27)$.

$$
\begin{aligned}
& 3 C_{3}^{(1,1)}=\left\{b a, b a^{\prime}, b a^{2} a^{\prime 2}\right\}, \\
& 3 C_{3}^{(1,2)}=\left\{b a^{2}, b a a^{\prime}, b a^{\prime 2}\right\}, \\
& 3 C_{3}^{(2,0)}=\left\{b^{2}, b^{2} a a^{\prime 2}, b^{2} a^{2} a^{\prime}\right\}, \\
& 3 C_{3}^{(2,1)}=\left\{b^{2} a, b^{2} a^{\prime}, b^{2} a^{2} a^{\prime 2}\right\}, \\
& 3 C_{3}^{(2,2)}=\left\{b^{2} a^{2}, b^{2} a a^{\prime}, b^{2} a^{\prime 2}\right\} .
\end{aligned}
$$

The $\Delta(27)$ has nine one-dimensional representations, which we denote as $\mathbf{1}_{k, r}(k, r=$ $0,1,2)$, and two three-dimensional irreducible representations $\mathbf{3}$ and $\overline{\mathbf{3}}$. The explicit form of the group generators in each irreducible representation is

$$
\begin{aligned}
& \mathbf{1}_{k, r}: a=\omega^{r}, \quad a^{\prime}=\omega^{r} \quad b=\omega^{k}, \\
& \text { 3: } \quad a=\left(\begin{array}{ccc}
\omega & 0 & 0 \\
0 & 1 & 0 \\
0 & 0 & \omega^{2}
\end{array}\right), \quad a^{\prime}=\left(\begin{array}{ccc}
\omega^{2} & 0 & 0 \\
0 & \omega & 0 \\
0 & 0 & 1
\end{array}\right), \quad b=\left(\begin{array}{lll}
0 & 1 & 0 \\
0 & 0 & 1 \\
1 & 0 & 0
\end{array}\right) \text {, } \\
& \overline{3}: \quad a=\left(\begin{array}{ccc}
\omega^{2} & 0 & 0 \\
0 & 1 & 0 \\
0 & 0 & \omega
\end{array}\right), \quad a^{\prime}=\left(\begin{array}{ccc}
\omega & 0 & 0 \\
0 & \omega^{2} & 0 \\
0 & 0 & 1
\end{array}\right), \quad b=\left(\begin{array}{lll}
0 & 1 & 0 \\
0 & 0 & 1 \\
1 & 0 & 0
\end{array}\right) \text {, }
\end{aligned}
$$

where $\omega=e^{2 \pi i / 3}$ is the cube root of unity. Notice that $\mathbf{3}$ and $\overline{\mathbf{3}}$ are complex representations dual to each other. From the character table of the group, shown in table 6, we can straightforwardly obtain the Kronecker products between the various representations

$$
\begin{aligned}
& \mathbf{1}_{k, r} \otimes \mathbf{1}_{k^{\prime}, r^{\prime}}=\mathbf{1}_{\left[k+k^{\prime}\right],\left[r+r^{\prime}\right]}, \quad \mathbf{3} \otimes \mathbf{1}_{k, r}=\mathbf{3}, \quad \overline{\mathbf{3}} \otimes \mathbf{1}_{k, r}=\overline{\mathbf{3}}, \\
& \mathbf{3} \otimes \overline{\mathbf{3}}=\sum_{k, r=0}^{2} \mathbf{1}_{k, r}, \quad \mathbf{3} \otimes \mathbf{3}=\overline{\mathbf{3}} \oplus \overline{\mathbf{3}} \oplus \overline{\mathbf{3}}, \quad \overline{\mathbf{3}} \otimes \overline{\mathbf{3}}=\mathbf{3} \oplus \mathbf{3} \oplus \mathbf{3},
\end{aligned}
$$


where $[n]$ stands for $n$ mod 3, whenever $n$ is an integer. Starting from the representation matrices of the generators in different irreducible representations, we can calculate the ClebschGordan (CG) coefficients for the Kronecker products listed above. All CG coefficients are presented in the form $\alpha \otimes \beta$, where $\alpha_{i}$ stands for the elements of the first representation and $\beta_{j}$ those of the second one. In the following, we adopt the convention $\alpha_{[3]}=\alpha_{0} \equiv \alpha_{3}$.

- $\mathbf{1}_{k, r} \otimes \mathbf{1}_{k^{\prime}, r^{\prime}}=\mathbf{1}_{\left[k+k^{\prime}\right],\left[r+r^{\prime}\right]}$

$$
\left(\alpha_{1}\right)_{\mathbf{1}_{k, r}} \otimes\left(\beta_{1}\right)_{\mathbf{1}_{k^{\prime}, r^{\prime}}}=\left(\alpha_{1} \beta_{1}\right)_{\mathbf{1}_{\left[k+k^{\prime}\right],\left[r+r^{\prime}\right]}} .
$$

- $\mathbf{3} \otimes \mathbf{1}_{k, r}=\mathbf{3}$

$$
\left(\begin{array}{l}
\alpha_{1} \\
\alpha_{2} \\
\alpha_{3}
\end{array}\right)_{\mathbf{3}} \otimes\left(\beta_{1}\right)_{\mathbf{1}_{k, r}}=\left(\begin{array}{c}
\alpha_{[1+r]} \beta_{1} \\
\omega^{k} \alpha_{[2+r]} \beta_{1} \\
\omega^{2 k} \alpha_{[3+r]} \beta_{1}
\end{array}\right)_{\mathbf{3}}
$$

- $\overline{\mathbf{3}} \otimes \mathbf{1}_{k, r}=\overline{\mathbf{3}}$

$$
\left(\begin{array}{c}
\alpha_{1} \\
\alpha_{2} \\
\alpha_{3}
\end{array}\right)_{\overline{\mathbf{3}}} \otimes\left(\beta_{1}\right)_{\mathbf{1}_{k, r}}=\left(\begin{array}{c}
\alpha_{[1-r]} \beta_{1} \\
\omega^{k} \alpha_{[2-r]} \beta_{1} \\
\omega^{2 k} \alpha_{[3-r]} \beta_{1}
\end{array}\right)_{\overline{\mathbf{3}}}
$$

- $\mathbf{3} \otimes \overline{\mathbf{3}}=\sum_{k, r=0}^{2} \mathbf{1}_{k, r}$

$$
\begin{aligned}
& \left(\begin{array}{l}
\alpha_{1} \\
\alpha_{2} \\
\alpha_{3}
\end{array}\right)_{\mathbf{3}} \otimes\left(\begin{array}{l}
\beta_{1} \\
\beta_{2} \\
\beta_{3}
\end{array}\right)_{\overline{\mathbf{3}}} \\
& =\left(\alpha_{1} \beta_{1}+\alpha_{2} \beta_{2}+\alpha_{3} \beta_{3}\right)_{\mathbf{1}_{0,0}} \oplus\left(\alpha_{1} \beta_{1}+\omega^{2} \alpha_{2} \beta_{2}+\omega \alpha_{3} \beta_{3}\right)_{\mathbf{1}_{1,0}} \oplus\left(\alpha_{1} \beta_{1}+\omega \alpha_{2} \beta_{2}+\omega^{2} \alpha_{3} \beta_{3}\right)_{\mathbf{1}_{2,0}} \\
& \oplus\left(\alpha_{3} \beta_{1}+\alpha_{1} \beta_{2}+\alpha_{2} \beta_{3}\right)_{\mathbf{1}_{0,1}} \oplus\left(\alpha_{3} \beta_{1}+\omega^{2} \alpha_{1} \beta_{2}+\omega \alpha_{2} \beta_{3}\right)_{\mathbf{1}_{1,1}} \oplus\left(\alpha_{3} \beta_{1}+\omega \alpha_{1} \beta_{2}+\omega^{2} \alpha_{2} \beta_{3}\right)_{\mathbf{1}_{2,1}} \\
& \oplus\left(\alpha_{2} \beta_{1}+\alpha_{3} \beta_{2}+\alpha_{1} \beta_{3}\right)_{\mathbf{1}_{0,2}} \oplus\left(\alpha_{2} \beta_{1}+\omega^{2} \alpha_{3} \beta_{2}+\omega \alpha_{1} \beta_{3}\right)_{\mathbf{1}_{1,2}} \oplus\left(\alpha_{2} \beta_{1}+\omega \alpha_{3} \beta_{2}+\omega^{2} \alpha_{1} \beta_{3}\right)_{\mathbf{1}_{2,2}} .
\end{aligned}
$$

- $\mathbf{3} \otimes \mathbf{3}=\overline{\mathbf{3}}_{S_{1}} \oplus \overline{\mathbf{3}}_{S_{2}} \oplus \overline{\mathbf{3}}_{A}$

$$
\left(\begin{array}{l}
\alpha_{1} \\
\alpha_{2} \\
\alpha_{3}
\end{array}\right)_{\mathbf{3}} \otimes\left(\begin{array}{l}
\beta_{1} \\
\beta_{2} \\
\beta_{3}
\end{array}\right)_{\mathbf{3}}=\left(\begin{array}{l}
\alpha_{1} \beta_{1} \\
\alpha_{2} \beta_{2} \\
\alpha_{3} \beta_{3}
\end{array}\right)_{\overline{\mathbf{3}}_{S_{1}}} \oplus \frac{1}{2}\left(\begin{array}{c}
\alpha_{2} \beta_{3}+\alpha_{3} \beta_{2} \\
\alpha_{3} \beta_{1}+\alpha_{1} \beta_{3} \\
\alpha_{1} \beta_{2}+\alpha_{2} \beta_{1}
\end{array}\right)_{\overline{\mathbf{3}}_{S_{2}}} \oplus \frac{1}{2}\left(\begin{array}{c}
\alpha_{2} \beta_{3}-\alpha_{3} \beta_{2} \\
\alpha_{3} \beta_{1}-\alpha_{1} \beta_{3} \\
\alpha_{1} \beta_{2}-\alpha_{2} \beta_{1}
\end{array}\right)_{\overline{\mathbf{3}}_{A}},
$$

where the subscripts " $S$ " and " $A$ " denote symmetric and anti-symmetric combinations respectively. 
- $\overline{\mathbf{3}} \otimes \overline{\mathbf{3}}=\mathbf{3}_{S_{1}} \oplus \mathbf{3}_{S_{2}} \oplus \mathbf{3}_{A}$

$$
\left(\begin{array}{l}
\alpha_{1} \\
\alpha_{2} \\
\alpha_{3}
\end{array}\right)_{\overline{3}} \otimes\left(\begin{array}{l}
\beta_{1} \\
\beta_{2} \\
\beta_{3}
\end{array}\right)_{\overline{3}}=\left(\begin{array}{l}
\alpha_{1} \beta_{1} \\
\alpha_{2} \beta_{2} \\
\alpha_{3} \beta_{3}
\end{array}\right)_{\mathbf{3}_{S_{1}}} \oplus \frac{1}{2}\left(\begin{array}{c}
\alpha_{2} \beta_{3}+\alpha_{3} \beta_{2} \\
\alpha_{3} \beta_{1}+\alpha_{1} \beta_{3} \\
\alpha_{1} \beta_{2}+\alpha_{2} \beta_{1}
\end{array}\right)_{\mathbf{3}_{S_{2}}} \oplus \frac{1}{2}\left(\begin{array}{l}
\alpha_{2} \beta_{3}-\alpha_{3} \beta_{2} \\
\alpha_{3} \beta_{1}-\alpha_{1} \beta_{3} \\
\alpha_{1} \beta_{2}-\alpha_{2} \beta_{1}
\end{array}\right)_{\mathbf{3}_{A}} .
$$

\section{Vacuum alignment}

In this appendix, we shall investigate the problem of achieving the vacuum configuration in eq. (2.10) and eq. (2.11). For self-consistency, all flavon fields $\varphi, \xi, \sigma_{1}$ and $\sigma_{2}$ are treated as complex, given the form of the $\Delta(27)$ representation matrices, and the fact that the $Z_{4}$ charge of $\sigma_{2}$ is purely imaginary. Since the flavons $\varphi$ and $\xi, \sigma_{1}, \sigma_{2}$ are assumed to be localized at $y=0$ and $y=L$ respectively, the vacuum alignment problem is greatly simplified. At the UV brane $y=0$, the flavon $\varphi$ transforms in the manner listed in table 1 . The scalar potential invariant under the flavor symmetry $\Delta(27) \otimes Z_{4} \otimes Z_{4}^{\prime}$ can be written as:

$$
\begin{aligned}
V_{\mathrm{UV}}(\varphi)= & M_{\varphi}^{2}\left(\varphi \varphi^{*}\right) \mathbf{1}_{0,0}+f_{1}\left[(\varphi \varphi)_{\overline{\mathbf{3}}_{S_{1}}}\left(\varphi^{*} \varphi^{*}\right)_{\mathbf{3}_{S_{1}}}\right]_{\mathbf{1}_{0,0}}+f_{2}\left[(\varphi \varphi)_{\overline{\mathbf{3}}_{S_{2}}}\left(\varphi^{*} \varphi^{*}\right)_{\mathbf{3}_{S_{2}}}\right]_{\mathbf{1}_{0,0}} \\
& +f_{3}\left[(\varphi \varphi)_{\overline{\mathbf{3}}_{S_{1}}}\left(\varphi^{*} \varphi^{*}\right)_{\mathbf{3}_{S_{2}}}\right]_{\mathbf{1}_{0,0}}+f_{3}^{*}\left[(\varphi \varphi)_{\overline{\mathbf{3}}_{S_{2}}}\left(\varphi^{*} \varphi^{*}\right)_{\mathbf{3}_{S_{1}}}\right]_{\mathbf{1}_{0,0}}
\end{aligned}
$$

with real couplings $M_{\varphi}^{2}, f_{1}$ and $f_{2}$. Note that $\varphi=\left(\varphi_{1}, \varphi_{2}, \varphi_{3}\right)$ is a $\Delta(27)$ triplet $\mathbf{3}$, and its complex conjugate $\varphi^{*}=\left(\varphi_{1}^{*}, \varphi_{2}^{*}, \varphi_{3}^{*}\right)$ transforms consequently as $\overline{\mathbf{3}}$. Focusing on the field configuration

$$
\langle\varphi\rangle=(1,1,1) v_{\varphi}
$$

the minimum conditions for the UV potential read

$$
\frac{\partial V_{\mathrm{UV}}(\varphi)}{\partial \varphi_{1}^{*}}=\frac{\partial V_{\mathrm{UV}}(\varphi)}{\partial \varphi_{2}^{*}}=\frac{\partial V_{\mathrm{UV}}(\varphi)}{\partial \varphi_{3}^{*}}=v_{\varphi}\left[M_{\varphi}^{2}+2\left(f_{1}+f_{2}+f_{3}+f_{3}^{*}\right)\left|v_{\varphi}\right|^{2}\right]=0,
$$

leading to a non zero solution

$$
\left|v_{\varphi}\right|^{2}=-\frac{M_{\varphi}^{2}}{2\left(f_{1}+f_{2}+f_{3}+f_{3}^{*}\right)},
$$

that holds in a finite portion of parameter space with $f_{1}+f_{2}+f_{3}+f_{3}^{*}<0$.

Similarly, at the IR brane $y=L$, the most general renormalizable scalar potential $V_{\mathrm{IR}}$ involving the flavon fields $\xi, \sigma_{1}, \sigma_{2}$ is

$$
\begin{aligned}
V_{\mathrm{IR}}\left(\xi, \sigma_{1}, \sigma_{2}\right)= & M_{\xi}^{2}\left(\xi \xi^{*}\right)_{\mathbf{1}_{0,0}}+M_{\sigma_{1}}^{2}\left(\sigma_{1} \sigma_{1}^{*}\right)_{\mathbf{1}_{0,0}}+M_{\sigma_{2}}^{2}\left(\sigma_{2} \sigma_{2}^{*}\right)_{\mathbf{1}_{0,0}}+g_{1}\left[(\xi \xi)_{\overline{\mathbf{3}}_{S_{1}}}\left(\xi^{*} \xi^{*}\right)_{\mathbf{3}_{S_{1}}}\right]_{\mathbf{1}_{0,0}} \\
& +g_{2}\left[(\xi \xi)_{\overline{\mathbf{3}}_{S_{2}}}\left(\xi^{*} \xi^{*}\right)_{\mathbf{3}_{S_{2}}}\right]_{\mathbf{1}_{0,0}}+g_{3}\left[(\xi \xi)_{\overline{\mathbf{3}}_{S_{1}}}\left(\xi^{*} \xi^{*}\right)_{\mathbf{3}_{S_{2}}}\right]_{\mathbf{1}_{0,0}}+g_{3}^{*}\left[(\xi \xi)_{\overline{\mathbf{3}}_{S_{2}}}\left(\xi^{*} \xi^{*}\right)_{\mathbf{3}_{S_{1}}}\right]_{\mathbf{1}_{0,0}} \\
& +g_{4} \sigma_{1}^{2} \sigma_{1}^{* 2}+g_{5} \sigma_{2}^{2} \sigma_{2}^{* 2}+g_{6}\left|\sigma_{1}\right|^{2}\left|\sigma_{2}\right|^{2}+g_{7}\left(\xi \xi^{*}\right) \mathbf{1}_{\mathbf{1}_{0,0}}\left|\sigma_{1}\right|^{2}+g_{8}\left(\xi \xi^{*}\right)_{\mathbf{1}_{0,0}}\left|\sigma_{2}\right|^{2} \\
& +g_{9}\left(\xi \xi^{*}\right)_{\mathbf{1}_{0,1}} \sigma_{1}^{2}+g_{9}^{*}\left(\xi \xi^{*}\right)_{\mathbf{1}_{0,2}} \sigma_{1}^{* 2}
\end{aligned}
$$


where all couplings, excluding $g_{3}$ and $g_{9}$, are real. For this potential, the Case I alignment

$$
\langle\xi\rangle=\left(0, v_{\xi}, 0\right), \quad\left\langle\sigma_{1}\right\rangle=v_{\sigma_{1}}, \quad\left\langle\sigma_{2}\right\rangle=v_{\sigma_{2}},
$$

determines the minimization conditions

$$
\begin{aligned}
& \frac{\partial V_{\mathrm{IR}}\left(\xi, \sigma_{1}, \sigma_{2}\right)}{\partial \xi_{1}^{*}}=g_{9}^{*} v_{\xi} v_{\sigma_{1}}^{* 2}=0, \\
& \frac{\partial V_{\mathrm{IR}}\left(\xi, \sigma_{1}, \sigma_{2}\right)}{\partial \xi_{2}^{*}}=v_{\xi}\left(M_{\xi}^{2}+2 g_{1}\left|v_{\xi}\right|^{2}+g_{7}\left|v_{\sigma_{1}}\right|^{2}+g_{8}\left|v_{\sigma_{2}}\right|^{2}\right)=0, \\
& \frac{\partial V_{\mathrm{IR}}\left(\xi, \sigma_{1}, \sigma_{2}\right)}{\partial \xi_{3}^{*}}=g_{9} v_{\xi} v_{\sigma_{1}}^{2}=0, \\
& \frac{\partial V_{\mathrm{IR}}\left(\xi, \sigma_{1}, \sigma_{2}\right)}{\partial \sigma_{1}^{*}}=v_{\sigma_{1}}\left(M_{\sigma_{1}}^{2}+2 g_{4}\left|v_{\sigma_{1}}\right|^{2}+g_{7}\left|v_{\xi}\right|^{2}+g_{6}\left|v_{\sigma_{2}}\right|^{2}\right)=0, \\
& \frac{\partial V_{\mathrm{IR}}\left(\xi, \sigma_{1}, \sigma_{2}\right)}{\partial \sigma_{2}^{*}}=v_{\sigma_{2}}\left(M_{\sigma_{2}}^{2}+2 g_{5}\left|v_{\sigma_{2}}\right|^{2}+g_{8}\left|v_{\xi}\right|^{2}+g_{6}\left|v_{\sigma_{1}}\right|^{2}\right)=0 .
\end{aligned}
$$

From the above equations, it is clear that non-trivial solutions in this sector are only achievable by fine tuning the $g_{9}$ parameter to satisfy $g_{9}=0$. This choice can be enforced by an additional dynamical mechanism capable of switching off the $\left(\xi \xi^{*}\right)_{\mathbf{1}_{0,1}} \sigma_{1}^{2}$ and $\left(\xi \xi^{*}\right)_{\mathbf{1}_{0,2}} \sigma_{1}^{* 2}$ terms in the potential. Such scenario could be naturally realized in a supersymmetric extension $[20,59]$. As this possibility lies beyond the scope or the present work, we simply impose the condition $g_{9}=0$ in the general potential. Then, the obtained solutions are given by

$$
\begin{aligned}
\left|v_{\xi}\right|^{2} & =\frac{\left(g_{6}^{2}-4 g_{4} g_{5}\right) M_{\xi}^{2}+\left(2 g_{5} g_{7}-g_{6} g_{8}\right) M_{\sigma_{1}}^{2}+\left(2 g_{4} g_{8}-g_{6} g_{7}\right) M_{\sigma_{2}}^{2}}{2\left(4 g_{1} g_{4} g_{5}+g_{6} g_{7} g_{8}-g_{1} g_{6}^{2}-g_{4} g_{8}^{2}-g_{5} g_{7}^{2}\right)}, \\
\left|v_{\sigma_{1}}\right|^{2} & =\frac{\left(2 g_{5} g_{7}-g_{6} g_{8}\right) M_{\xi}^{2}+\left(g_{8}^{2}-4 g_{1} g_{5}\right) M_{\sigma_{1}}^{2}+\left(2 g_{1} g_{6}-g_{7} g_{8}\right) M_{\sigma_{2}}^{2}}{2\left(4 g_{1} g_{4} g_{5}+g_{6} g_{7} g_{8}-g_{1} g_{6}^{2}-g_{4} g_{8}^{2}-g_{5} g_{7}^{2}\right)}, \\
\left|v_{\sigma_{2}}\right|^{2} & =\frac{\left(2 g_{4} g_{8}-g_{6} g_{7}\right) M_{\xi}^{2}+\left(2 g_{1} g_{6}-g_{7} g_{8}\right) M_{\sigma_{1}}^{2}+\left(g_{7}^{2}-4 g_{1} g_{4}\right) M_{\sigma_{2}}^{2}}{2\left(4 g_{1} g_{4} g_{5}+g_{6} g_{7} g_{8}-g_{1} g_{6}^{2}-g_{4} g_{8}^{2}-g_{5} g_{7}^{2}\right)} .
\end{aligned}
$$

The right-handed side of these expressions can be positive in a finite region of parameter space. Analogously, for the Case II vacuum configuration

$$
\langle\xi\rangle=(1, \omega, 1) v_{\xi}, \quad\left\langle\sigma_{1}\right\rangle=v_{\sigma_{1}}, \quad\left\langle\sigma_{2}\right\rangle=v_{\sigma_{2}},
$$

the minimization conditions are

$$
\begin{aligned}
& \frac{\partial V_{\mathrm{IR}}\left(\xi, \sigma_{1}, \sigma_{2}\right)}{\partial \xi_{1}^{*}}=v_{\xi}\left[M_{\xi}^{2}+2\left(g_{1}+g_{2}+\omega^{2} g_{3}+\omega g_{3}^{*}\right)\left|v_{\xi}\right|^{2}+g_{7}\left|v_{\sigma_{1}}\right|^{2}+g_{8}\left|v_{\sigma_{2}}\right|^{2}+g_{9} v_{\sigma_{1}}^{2}+\omega g_{9}^{*} v_{\sigma_{1}}^{* 2}\right]=0, \\
& \frac{\partial V_{\mathrm{IR}}\left(\xi, \sigma_{1}, \sigma_{2}\right)}{\partial \xi_{2}^{*}}=\omega v_{\xi}\left[M_{\xi}^{2}+2\left(g_{1}+g_{2}+\omega^{2} g_{3}+\omega g_{3}^{*}\right)\left|v_{\xi}\right|^{2}+g_{7}\left|v_{\sigma_{1}}\right|^{2}+g_{8}\left|v_{\sigma_{2}}\right|^{2}+\omega^{2} g_{9} v_{\sigma_{1}}^{2}+\omega^{2} g_{9}^{*} v_{\sigma_{1}}^{* 2}\right]=0, \\
& \frac{\partial V_{\mathrm{IR}}\left(\xi, \sigma_{1}, \sigma_{2}\right)}{\partial \xi_{3}^{*}}=v_{\xi}\left[M_{\xi}^{2}+2\left(g_{1}+g_{2}+\omega^{2} g_{3}+\omega g_{3}^{*}\right)\left|v_{\xi}\right|^{2}+g_{7}\left|v_{\sigma_{1}}\right|^{2}+g_{8}\left|v_{\sigma_{2}}\right|^{2}+\omega g_{9} v_{\sigma_{1}}^{2}+g_{9}^{*} v_{\sigma_{1}}^{* 2}\right]=0, \\
& \frac{\partial V_{\mathrm{IR}}\left(\xi, \sigma_{1}, \sigma_{2}\right)}{\partial \sigma_{1}^{*}}=v_{\sigma_{1}}\left[M_{\sigma_{1}}^{2}+2 g_{4}\left|v_{\sigma_{1}}\right|^{2}+g_{6}\left|v_{\sigma_{2}}\right|^{2}+3 g_{7}\left|v_{\xi}\right|^{2}\right]=0, \\
& \frac{\partial V_{\mathrm{IR}}\left(\xi, \sigma_{1}, \sigma_{2}\right)}{\partial \sigma_{2}^{*}}=v_{\sigma_{2}}\left[M_{\sigma_{2}}^{2}+2 g_{5}\left|v_{\sigma_{2}}\right|^{2}+g_{6}\left|v_{\sigma_{1}}\right|^{2}+3 g_{8}\left|v_{\xi}\right|^{2}\right]=0 .
\end{aligned}
$$


Again, these equations are incompatible unless $g_{9}=0$. Once the coupling $g_{9}$ is enforced to vanish, we are left with three independent linear equations for the three unknown variables $v_{\xi}, v_{\sigma_{1}}$ and $v_{\sigma_{2}}$. The solutions can be easily found as

$$
\begin{aligned}
\left|v_{\xi}\right|^{2} & =\frac{\left(4 g_{4} g_{5}-g_{6}^{2}\right) M_{\xi}^{2}+\left(g_{6} g_{8}-2 g_{5} g_{7}\right) M_{\sigma_{1}}^{2}+\left(g_{6} g_{7}-2 g_{4} g_{8}\right) M_{\sigma_{2}}^{2}}{2 \widetilde{g}\left(g_{6}^{2}-4 g_{4} g_{5}\right)+6\left(g_{4} g_{8}^{2}+g_{5} g_{7}^{2}-g_{6} g_{7} g_{8}\right)}, \\
\left|v_{\sigma_{1}}\right|^{2} & =\frac{\left(3 g_{6} g_{8}-6 g_{5} g_{7}\right) M_{\xi}^{2}+\left(4 \widetilde{g} g_{5}-3 g_{8}^{2}\right) M_{\sigma_{1}}^{2}+\left(3 g_{7} g_{8}-2 \widetilde{g} g_{6}\right) M_{\sigma_{2}}^{2}}{2 \widetilde{g}\left(g_{6}^{2}-4 g_{4} g_{5}\right)+6\left(g_{4} g_{8}^{2}+g_{5} g_{7}^{2}-g_{6} g_{7} g_{8}\right)}, \\
\left|v_{\sigma_{2}}\right|^{2} & =\frac{\left(3 g_{6} g_{7}-6 g_{4} g_{8}\right) M_{\xi}^{2}+\left(3 g_{7} g_{8}-2 \widetilde{g} g_{6}\right) M_{\sigma_{1}}^{2}+\left(4 \widetilde{g} g_{4}-3 g_{7}^{2}\right) M_{\sigma_{2}}^{2}}{2 \widetilde{g}\left(g_{6}^{2}-4 g_{4} g_{5}\right)+6\left(g_{4} g_{8}^{2}+g_{5} g_{7}^{2}-g_{6} g_{7} g_{8}\right)} .
\end{aligned}
$$

where we have defined $\widetilde{g} \equiv g_{1}+g_{2}+\omega^{2} g_{3}+\omega g_{3}^{*}$. Therefore, both $\langle\xi\rangle=\left(0, v_{\xi}, 0\right)$ and $\langle\xi\rangle=(1, \omega, 1) v_{\xi}$ alignments can describe the local minimum of $V_{\mathrm{IR}}\left(\xi, \sigma_{1}, \sigma_{2}\right)$, depending on the parameter values. In the case of $g_{1} \ll \widetilde{g}$, the $\operatorname{VEV}\langle\xi\rangle=\left(0, v_{\xi}, 0\right)$ is preferred over $\langle\xi\rangle=(1, \omega, 1) v_{\xi}$, while $V_{\mathrm{IR}}\left(\xi, \sigma_{1}, \sigma_{2}\right)$ is minimized by $\langle\xi\rangle=(1, \omega, 1) v_{\xi}$ for $g_{1} \gg \widetilde{g}$.

Open Access. This article is distributed under the terms of the Creative Commons Attribution License (CC-BY 4.0), which permits any use, distribution and reproduction in any medium, provided the original author(s) and source are credited.

\section{References}

[1] Particle Data Group collaboration, K.A. Olive et al., Review of particle physics, Chin. Phys. C 38 (2014) 090001 [inSPIRE].

[2] K.S. Babu, E. Ma and J.W.F. Valle, Underlying $A_{4}$ symmetry for the neutrino mass matrix and the quark mixing matrix, Phys. Lett. B 552 (2003) 207 [hep-ph/0206292] [INSPIRE].

[3] S. Morisi and J.W.F. Valle, Neutrino masses and mixing: a flavour symmetry roadmap, Fortsch. Phys. 61 (2013) 466 [arXiv:1206.6678] [INSPIRE].

[4] G. Altarelli, F. Feruglio, L. Merlo and E. Stamou, Discrete flavour groups, $\theta_{13}$ and lepton flavour violation, JHEP 08 (2012) 021 [arXiv:1205.4670] [INSPIRE].

[5] R.M. Fonseca and W. Grimus, Classification of lepton mixing patterns from finite flavour symmetries, arXiv:1410.4133 [INSPIRE].

[6] G. Altarelli and F. Feruglio, Discrete flavor symmetries and models of neutrino mixing, Rev. Mod. Phys. 82 (2010) 2701 [arXiv:1002.0211] [INSPIRE].

[7] H. Ishimori, T. Kobayashi, H. Ohki, Y. Shimizu, H. Okada and M. Tanimoto, Non-Abelian discrete symmetries in particle physics, Prog. Theor. Phys. Suppl. 183 (2010) 1 [arXiv: 1003.3552] [INSPIRE].

[8] S.F. King and C. Luhn, Neutrino mass and mixing with discrete symmetry, Rept. Prog. Phys. 76 (2013) 056201 [arXiv:1301.1340] [InSPIRE].

[9] S.F. King, A. Merle, S. Morisi, Y. Shimizu and M. Tanimoto, Neutrino mass and mixing: from theory to experiment, New J. Phys. 16 (2014) 045018 [arXiv:1402.4271] [InSPIRE].

[10] P.F. Harrison, D.H. Perkins and W.G. Scott, Tri-bimaximal mixing and the neutrino oscillation data, Phys. Lett. B 530 (2002) 167 [hep-ph/0202074] [INSPIRE]. 
[11] DaYa Bay collaboration, F.P. An et al., Observation of electron-antineutrino disappearance at Daya Bay, Phys. Rev. Lett. 108 (2012) 171803 [arXiv:1203.1669] [INSPIRE].

[12] T2K collaboration, K. Abe et al., Indication of electron neutrino appearance from an accelerator-produced off-axis muon neutrino beam, Phys. Rev. Lett. 107 (2011) 041801 [arXiv:1106.2822] [INSPIRE].

[13] MINOS collaboration, P. Adamson et al., Measurement of neutrino and antineutrino oscillations using beam and atmospheric data in MINOS, Phys. Rev. Lett. 110 (2013) 251801 [arXiv: 1304.6335] [INSPIRE].

[14] RENO collaboration, J.K. Ahn et al., Observation of reactor electron antineutrino disappearance in the RENO experiment, Phys. Rev. Lett. 108 (2012) 191802 [arXiv: 1204.0626] [INSPIRE].

[15] S. Morisi, D.V. Forero, J.C. Romão and J.W.F. Valle, Neutrino mixing with revamped $A_{4}$ flavor symmetry, Phys. Rev. D 88 (2013) 016003 [arXiv: 1305.6774] [INSPIRE].

[16] S.M. Boucenna, S. Morisi, M. Tortola and J.W.F. Valle, Bi-large neutrino mixing and the Cabibbo angle, Phys. Rev. D 86 (2012) 051301 [arXiv:1206.2555] [INSPIRE].

[17] G.-J. Ding, S. Morisi and J.W.F. Valle, Bilarge neutrino mixing and Abelian flavor symmetry, Phys. Rev. D 87 (2013) 053013 [arXiv:1211.6506] [INSPIRE].

[18] S. Roy and N.N. Singh, Bi-large neutrino mixing with charged lepton correction, Indian J. Phys. 88 (2014) 513 [arXiv:1211.7207] [INSPIRE].

[19] L. Randall and R. Sundrum, A large mass hierarchy from a small extra dimension, Phys. Rev. Lett. 83 (1999) 3370 [hep-ph/9905221] [INSPIRE].

[20] G. Altarelli and F. Feruglio, Tri-bimaximal neutrino mixing from discrete symmetry in extra dimensions, Nucl. Phys. B 720 (2005) 64 [hep-ph/0504165] [INSPIRE].

[21] G. Altarelli, F. Feruglio and C. Hagedorn, A SUSY SU(5) grand unified model of tri-bimaximal mixing from $A_{4}$, JHEP 03 (2008) 052 [arXiv: 0802.0090] [INSPIRE].

[22] T.J. Burrows and S.F. King, $A_{4}$ family symmetry from SU(5) SUSY GUTs in $6 d$, Nucl. Phys. B 835 (2010) 174 [arXiv:0909.1433] [INSPIRE].

[23] C. Csáki, C. Delaunay, C. Grojean and Y. Grossman, A model of lepton masses from a warped extra dimension, JHEP 10 (2008) 055 [arXiv:0806.0356] [INSPIRE].

[24] M.-C. Chen, K.T. Mahanthappa and F. Yu, A viable Randall-Sundrum model for quarks and leptons with $T^{\prime}$ family symmetry, Phys. Rev. D 81 (2010) 036004 [arXiv:0907.3963] [INSPIRE].

[25] A. Kadosh and E. Pallante, An $A_{4}$ flavor model for quarks and leptons in warped geometry, JHEP 08 (2010) 115 [arXiv: 1004.0321] [InSPIRE].

[26] A. Kadosh and E. Pallante, CP violation and FCNC in a warped $A_{4}$ flavor model, JHEP 06 (2011) 121 [arXiv:1101.5420] [INSPIRE].

[27] A. Kadosh, $\Theta_{1} 3$ and charged lepton flavor violation in "warped" $A_{4}$ models, JHEP 06 (2013) 114 [arXiv:1303.2645] [INSPIRE].

[28] C. Alvarado, A. Aranda, O. Corradini, A.D. Rojas and E. Santos-Rodriguez, $Z_{4}$ flavor model in Randall-Sundrum model 1, Phys. Rev. D 86 (2012) 036010 [arXiv:1206.5216] [INSPIRE].

[29] G. von Gersdorff, M. Quirós and M. Wiechers, Neutrino mixing from Wilson lines in warped space, JHEP 02 (2013) 079 [arXiv:1208.4300] [INSPIRE]. 
[30] M. Frank, C. Hamzaoui, N. Pourtolami and M. Toharia, Unified flavor symmetry from warped dimensions, Phys. Lett. B 742 (2015) 178 [arXiv: 1406.2331] [INSPIRE].

[31] M. Frank, C. Hamzaoui, N. Pourtolami and M. Toharia, Fermion masses and mixing in general warped extra dimensional models, Phys. Rev. D 91 (2015) 116001 [arXiv: 1504.02780] [INSPIRE].

[32] F. del Aguila, A. Carmona and J. Santiago, Neutrino masses from an $A_{4}$ symmetry in holographic composite Higgs models, JHEP 08 (2010) 127 [arXiv:1001.5151] [INSPIRE].

[33] C. Hagedorn and M. Serone, General lepton mixing in holographic composite Higgs models, JHEP 02 (2012) 077 [arXiv:1110.4612] [INSPIRE].

[34] C. Hagedorn and M. Serone, Leptons in holographic composite Higgs models with non-Abelian discrete symmetries, JHEP 10 (2011) 083 [arXiv:1106.4021] [INSPIRE].

[35] D.V. Forero, M. Tortola and J.W.F. Valle, Neutrino oscillations refitted, Phys. Rev. D 90 (2014) 093006 [arXiv:1405.7540] [INSPIRE].

[36] G.-J. Ding and Y.-L. Zhou, Dirac neutrinos with $S_{4}$ flavor symmetry in warped extra dimensions, Nucl. Phys. B 876 (2013) 418 [arXiv:1304.2645] [INSPIRE].

[37] C. Csáki, J. Erlich and J. Terning, The effective Lagrangian in the Randall-Sundrum model and electroweak physics, Phys. Rev. D 66 (2002) 064021 [hep-ph/0203034] [inSPIRE].

[38] M. Carena, A. Delgado, E. Ponton, T.M.P. Tait and C.E.M. Wagner, Precision electroweak data and unification of couplings in warped extra dimensions, Phys. Rev. D 68 (2003) 035010 [hep-ph/0305188] [INSPIRE].

[39] J.A. Cabrer, G. von Gersdorff and M. Quirós, Suppressing electroweak precision observables in 5D warped models, JHEP 05 (2011) 083 [arXiv:1103.1388] [INSPIRE].

[40] J.A. Cabrer, G. von Gersdorff and M. Quirós, Improving naturalness in warped models with a heavy bulk Higgs boson, Phys. Rev. D 84 (2011) 035024 [arXiv:1104.3149] [INSPIRE].

[41] A. Carmona, E. Ponton and J. Santiago, Phenomenology of non-custodial warped models, JHEP 10 (2011) 137 [arXiv:1107.1500] [INSPIRE].

[42] P.R. Archer, M. Carena, A. Carmona and M. Neubert, Higgs production and decay in models of a warped extra dimension with a bulk Higgs, JHEP 01 (2015) 060 [arXiv:1408.5406] [INSPIRE].

[43] K. Agashe, A. Azatov and L. Zhu, Flavor violation tests of warped/composite SM in the two-site approach, Phys. Rev. D 79 (2009) 056006 [arXiv:0810.1016] [INSPIRE].

[44] P.R. Archer, S.J. Huber and S. Jager, Flavour physics in the soft wall model, JHEP 12 (2011) 101 [arXiv:1108.1433] [INSPIRE].

[45] J.A. Cabrer, G. von Gersdorff and M. Quirós, Flavor phenomenology in general $5 D$ warped spaces, JHEP 01 (2012) 033 [arXiv:1110.3324] [INSPIRE].

[46] K. Agashe, T. Okui and R. Sundrum, A common origin for neutrino anarchy and charged hierarchies, Phys. Rev. Lett. 102 (2009) 101801 [arXiv:0810.1277] [INSPIRE].

[47] P.R. Archer, The fermion mass hierarchy in models with warped extra dimensions and a bulk Higgs, JHEP 09 (2012) 095 [arXiv: 1204.4730] [INSPIRE].

[48] G. Cacciapaglia, C. Csáki, G. Marandella and J. Terning, The gaugephobic Higgs, JHEP 02 (2007) 036 [hep-ph/0611358] [INSPIRE]. 
[49] T. Gherghetta, A holographic view of beyond the Standard Model physics, arXiv:1008.2570 [INSPIRE].

[50] T. Gherghetta and A. Pomarol, Bulk fields and supersymmetry in a slice of AdS, Nucl. Phys. B 586 (2000) 141 [hep-ph/0003129] [INSPIRE].

[51] Y. Grossman and M. Neubert, Neutrino masses and mixings in nonfactorizable geometry, Phys. Lett. B 474 (2000) 361 [hep-ph/9912408] [INSPIRE].

[52] S.J. Huber and Q. Shafi, Neutrino oscillations and rare processes in models with a small extra dimension, Phys. Lett. B 512 (2001) 365 [hep-ph/0104293] [INSPIRE].

[53] I. de Medeiros Varzielas, S.F. King and G.G. Ross, Neutrino tri-bi-maximal mixing from a non-Abelian discrete family symmetry, Phys. Lett. B 648 (2007) 201 [hep-ph/0607045] [INSPIRE].

[54] E. Ma, Neutrino mass matrix from $\Delta(27)$ symmetry, Mod. Phys. Lett. A 21 (2006) 1917 [hep-ph/0607056] [INSPIRE].

[55] A. Aranda, C. Bonilla, S. Morisi, E. Peinado and J.W.F. Valle, Dirac neutrinos from flavor symmetry, Phys. Rev. D 89 (2014) 033001 [arXiv:1307.3553] [InSPIRE].

[56] G.C. Branco, J.M. Gerard and W. Grimus, Geometrical T violation, Phys. Lett. B 136 (1984) 383 [INSPIRE].

[57] G. Bhattacharyya, I. de Medeiros Varzielas and P. Leser, A common origin of fermion mixing and geometrical CP-violation and its test through Higgs physics at the LHC, Phys. Rev. Lett. 109 (2012) 241603 [arXiv:1210.0545] [INSPIRE].

[58] C. Jarlskog, Commutator of the quark mass matrices in the standard electroweak model and a measure of maximal CP-violation, Phys. Rev. Lett. 55 (1985) 1039 [INSPIRE].

[59] G. Altarelli and F. Feruglio, Tri-bimaximal neutrino mixing, $A_{4}$ and the modular symmetry, Nucl. Phys. B 741 (2006) 215 [hep-ph/0512103] [INSPIRE]. 